\title{
Power Scaling of Nonlinear Frequency Converted Tapered Diode Lasers for Biophotonics
}

Jensen, Ole Bjarlin; Hansen, Anders Kragh; Müller, A.; Sumpf, Bernd; Unterhuber, Angelika; Drexler, Wolfgang; Petersen, Paul Michael; Andersen, Peter E.

Published in:

I E E E Journal on Selected Topics in Quantum Electronics

Link to article, DOI:

10.1109/JSTQE.2013.2284423

Publication date:

2014

Document Version

Peer reviewed version

Link back to DTU Orbit

Citation (APA):

Jensen, O. B., Hansen, A. K., Müller, A., Sumpf, B., Unterhuber, A., Drexler, W., Petersen, P. M., \& Andersen, P. E. (2014). Power Scaling of Nonlinear Frequency Converted Tapered Diode Lasers for Biophotonics. I E E'E Journal on Selected Topics in Quantum Electronics, 20(2), [7100515]. https://doi.org/10.1109/JSTQE.2013.2284423

\section{General rights}

Copyright and moral rights for the publications made accessible in the public portal are retained by the authors and/or other copyright owners and it is a condition of accessing publications that users recognise and abide by the legal requirements associated with these rights.

- Users may download and print one copy of any publication from the public portal for the purpose of private study or research.

- You may not further distribute the material or use it for any profit-making activity or commercial gain

- You may freely distribute the URL identifying the publication in the public portal 


\title{
Power Scaling of Nonlinear Frequency Converted Tapered Diode Lasers for Biophotonics
}

\author{
Ole Bjarlin Jensen, Anders Kragh Hansen, André Müller, Bernd Sumpf, Angelika Unterhuber, \\ Wolfgang Drexler, Paul Michael Petersen, and Peter E. Andersen
}

\begin{abstract}
Diode lasers have proven to be versatile light sources for a wide range of applications. Nonlinear frequency conversion of high brightness diode lasers has recently resulted in visible light power levels in the watts range enabling an increasing number of applications within biophotonics. This review provides an overview of the developments within nonlinear frequency converted high power laser diodes in the visible spectral range. Single-pass nonlinear frequency doubling is presented as a non-sophisticated method to achieve watt-level output powers and possible routes to higher power and efficiency are included. Application examples within pumping of modelocked Ti:sapphire lasers and implementation of such lasers in optical coherence tomography are presented showing the application potential of these lasers.
\end{abstract}

Index Terms-Biophotonics, diode lasers, laser applications, nonlinear frequency conversion, nonlinear optics, optical coherence tomography, optical mixing, second harmonic generation, semiconductor lasers, sum frequency generation, Ti:sapphire lasers, ultrafast lasers.

\section{INTRODUCTION}

D iode lasers are highly efficient and compact light sources and as such they are used in a large range of applications [1], [2]. In contrast to lasers based on specific atomic transitions like solid state or gas lasers, diode laser material engineering allows the generation of light in a large part of the optical spectrum [3]. The large versatility of diode lasers allows the direct use within many biophotonics applications

Manuscript received XXXX.XX, 2013; revised XXXX XX, 2013; accepted XXXX XX, 2013. This work was supported in part by EU-FP6 integrated project WWW.BRIGHTER.EU contract IST-2005-035266 and European project FAMOS (FP7 ICT 317744). The authors acknowledge Heidelberg Engineering for equipment support.

O. B. Jensen, A. K. Hansen, P. M. Petersen and P. E. Andersen are with DTU Fotonik, Department of Photonics Engineering, Technical University of Denmark, Frederiksborgvej 399, 4000 Roskilde, Denmark (e-mail: ojen@ fotonik.dtu.dk; pape@ fotonik.dtu.dk; peta@ fotonik.dtu.dk).

A. Müller and B. Sumpf are with Ferdinand-Braun-Institut, Leibniz-Institut für Höchstfrequenztechnik, Gustav-Kirchhoff-Straße 4, 12489 Berlin, Germany (e-mail: andre.mueller@ fbh-berlin.de; bernd.sumpf@ fbh-berlin.de).

A. Unterhuber and W. Drexler are with Medical University of Vienna, Center for Medical Physics and Biomedical Engineering, Waehringer Guertel

18-20, AKH-Wien E4.L, 1090 Vienna, Austria (e-mail: angelika.unterhuber@meduniwien.ac.at;

wolfgang.drexler@meduniwien.ac.at).

Color versions of one or more of the figures in this paper are available online at http://ieeexplore.ieee.org.

Digital Object Identifier XXXXXXXXXXXXX like e.g. photocoagulation [4], diffuse optical imaging [5] and optical coherence tomography (OCT) [6], [7].

Until recently, it was not possible to obtain emission in the green-yellow spectral range from direct emitting diode lasers and the power levels are still limited to about $100 \mathrm{~mW}$ [8][12]. The green-yellow spectral range is particularly interesting for certain biophotonics applications as a main absorption peak of hemoglobin is located in the wavelength range 530-590 $\mathrm{nm}$ [13]. In diagnostics of various diseases, the broad spectrum and ultrashort pulses of Ti:sapphire lasers help to increase resolution of different imaging techniques such as multiphoton imaging [14]-[16] or OCT [17]. Ti:sapphire lasers are pumped in the green spectral range. Traditionally, frequency doubled diode pumped solid state lasers are the preferred light sources in applications requiring laser light in the green-yellow spectral region. These lasers show high output powers with excellent beam properties ideal for the applications. However, their physical dimensions, low efficiency and high cost limit widespread adoption of applications.

Nonlinear frequency conversion of diode lasers is an attractive method to circumvent some of the limitations of solid state lasers as outlined in the review article by Jechow et al. [18]. Diode lasers are compact and efficient and in high volumes they can be produced at very low cost. By tailoring the diode laser wavelength and the nonlinear crystal, many different applications can be addressed. For efficient frequency conversion, certain parameters like spectral and spatial quality must be optimized. With the introduction of high brightness tapered diode lasers, the beam quality of high power diode lasers has reached a level suitable for direct frequency conversion. Spectral narrowing of the emission from these tapered diode lasers has been implemented in different ways to enable efficient frequency conversion. External cavity techniques involving either diffraction gratings or Bragg gratings have been demonstrated to provide narrow spectral widths [19]-[23]. With the introduction of Bragg gratings in the ridge waveguide section of tapered diode lasers, these lasers are furthermore highly stable and robust against influences from the surroundings. Output power levels from these lasers have reached more than $12 \mathrm{~W}$ in the near infrared spectral range [24]-[26]. Frequency doubling of these lasers in periodically poled crystals has resulted in up to $2 \mathrm{~W}$ of output power in the green spectral range [27]-[29] and combining two of these lasers with subsequent sum frequency generation 
has resulted in close to $4 \mathrm{~W}$ of nearly diffraction limited green light [30]. These power levels meet the requirements for direct applications of lasers in treatment of vascular diseases and for pumping of Ti:sapphire lasers [31], where multi-Watt-level powers are necessary. Frequency doubled diode lasers have also been demonstrated in the yellow-orange spectral range although at significantly lower output power [32]-[34]. The achieved power levels are mainly limited by the available output power from the tapered diode lasers. In order to increase the visible output power level even further, new concepts are required.

In this paper, we review the development of nonlinear frequency converted high power tapered diode lasers and present different concepts to reach higher output power using combinations of single-pass arrangements. We investigate the methods of second harmonic generation and possibilities for power scaling using beam combination and frequency conversion and show new routes to even higher output power levels. Application of frequency doubled tapered diode lasers for pumping of mode-locked Ti:sapphire lasers as light source for in vivo OCT imaging of the retina and skin will be demonstrated.

\section{NONLINEAR FREQUENCY CONVERSION}

Nonlinear frequency conversion was first demonstrated in 1961 by Franken et al. [35], where second harmonic generation (SHG) was obtained in a quartz crystal illuminated by a ruby laser. This demonstration led to a rapid progress in nonlinear optical frequency conversion and the demonstration of sum frequency generation (SFG) [36], [37], difference frequency generation (DFG) [38] and optical parametric oscillation [39]. All of these processes are second order nonlinear processes and form the basis for all work described here. We will use second harmonic generation as an example in the paragraphs below. Second harmonic generation is a degenerate case of sum frequency generation and the photons generated have twice the energy of the incoming fundamental photons and thus half the wavelength as sketched in Fig. 1.

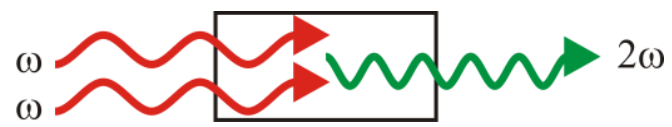

Fig. 1. Principle sketch of second harmonic generation. The generated photons have twice the energy and half the wavelength of the incoming fundamental photons.

In order to have efficient frequency conversion, two conditions must be satisfied - energy and momentum conservation [40], [41]. Energy conservation dictates the frequency of the generated photons. Momentum conservation, better known as phase matching, requires that the phase velocity is identical for all involved frequencies in order to have coherent addition throughout the length of the nonlinear material. The phase mismatch is given by

$$
\Delta k=k_{S H G}-2 k_{\text {Laser }}
$$

The power in the second harmonic beam depends strongly on the phase mismatch through the following relation assuming that the fundamental beam remains constant [40], [41]

$$
P_{S H G}=\eta_{S H G} P_{\text {Laser }}^{2} \operatorname{sinc}^{2}\left(\frac{\Delta k L}{2}\right)
$$

With $\eta_{S H G}$ as the nonlinear conversion efficiency and $P_{\text {Laser }}$ and $P_{S H G}$ the power of the fundamental and second harmonic beams, respectively. In order to have maximum efficiency, phase matching must be obtained implying $\Delta k=0$.

This requirement is only fulfilled when the refractive indices at the fundamental and second harmonic wavelengths are identical. Normal dispersion in most materials makes phase matching impossible to obtain except for the case of different polarizations in birefringent materials. Birefringent phase matching is only possible for a limited number of materials and only at certain wavelength regions. Typically a certain propagation angle in the nonlinear crystal is selected to achieve phase matching and the crystal temperature can also be used to change the refractive index of the crystal. When the propagation is at an angle with respect to the optical axes of the crystal, walk-off will occur, meaning that the light in the fundamental beam and the generated beam propagate in different directions lowering the conversion efficiency. A method circumventing this problem is quasi phase matching [42], [43], where periodic poling of the crystal compensates for the phase velocity mismatch, allowing phase matching at any wavelength and polarization combination of the incoming and generated waves. Furthermore, quasi phase matching gives access to the highest nonlinearity in many important materials and thus enables higher conversion efficiency. This is particularly important in single-pass frequency conversion, where the intensity of the fundamental light is limited. A typical curve that shows the SHG output power versus laser wavelength is shown in Fig. 2 using the expression in (2). This curve shows how the conversion efficiency depends strongly on the laser wavelength due to dispersion in the nonlinear crystal. For a $10 \mathrm{~mm}$ long periodically poled $\mathrm{LiNbO}_{3}$ crystal a wavelength acceptance bandwidth of $0.21 \mathrm{~nm}$ can be found for SHG of $1064 \mathrm{~nm}$ light. A narrow bandwidth laser is required in order to have efficient nonlinear frequency conversion. Similar results can be obtained by changing the propagation angle or crystal temperature.

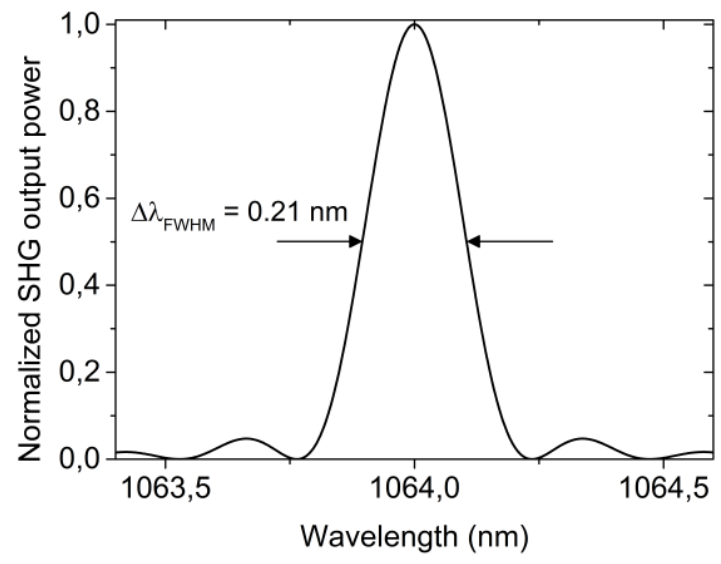

Fig. 2. Wavelength acceptance curve for SHG of a laser at $1064 \mathrm{~nm}$ in a 10 $\mathrm{mm}$ long periodically poled $\mathrm{MgO}: \mathrm{LiNbO}_{3}$ crystal poled with a period of 6.92 $\mu \mathrm{m}$.

Besides phase matching, the nonlinear conversion efficiency depends strongly on the intensity of the interacting fields. The higher the intensity, the more efficient is the conversion. It can 
be shown, however, that an optimum focusing condition of the fundamental wave exists that mainly depends on the crystal length [44].

The power of single-pass frequency doubled lasers is limited by the nonlinear conversion efficiency of the nonlinear crystals. A widely used method for increasing the conversion efficiency is by use of external enhancement cavities, where the fundamental power is enhanced to allow better conversion to the second harmonic beam. Numerous examples of cavity enhanced frequency doubled tapered diode lasers exist in the literature generating light in the ultraviolet and visible spectral ranges [45]-[50]. Frequency doubling in external enhancement cavities require the resonance frequency of the cavity to match the frequency of the input fundamental light, thus in practice requiring single-frequency operation of the laser. Active locking schemes are typically used to keep the cavity on resonance [51], [52] although all-passive approaches have been demonstrated [53]-[56]. Although external cavity frequency doubling is an efficient approach for increasing the conversion efficiency, the complexity and sensitivity to external perturbations makes this method less attractive. For this reason, external cavity frequency doubling will not be discussed further in this paper.

\section{TAPERED DIODE LASERS}

Diode lasers are the most efficient light sources available regarding electro-optical efficiency. In order to obtain high conversion efficiency in the nonlinear frequency conversion a high output power from the diode laser is desirable. One way to increase the maximum output power from diode lasers is to increase the facet width in order to lower the power density on the facet. Such lasers are referred to as broad area diode lasers. They can provide very high output powers in excess of $20 \mathrm{~W}$ [57]-[59], but they have one major drawback, which is their poor beam properties resulting in poor conversion efficiency in frequency conversion. External feedback might alleviate the problem, however, at the expense of increased complexity in terms of practical realization [60]-[63]. A better way to get around the poor beam quality and still obtain high output power is to use tapered diode laser structures shown in Fig. 3 (a). The tapered diode laser consists of a single-mode waveguide providing good beam quality and a tapered section amplifying the beam exiting the single-mode section [64], [65]. The taper angle is selected to fit the diffraction angle of the light emitted from the single-mode section in order to preserve the beam properties.

Tapered diode lasers emitting more than $10 \mathrm{~W}$ of output power have been demonstrated [24], [25], [66]. The beam properties of tapered diode lasers differ somewhat from the perfect Gaussian beam because of various effects in the tapered section of the laser. One important effect is that the carrier density gets depleted by the field propagating from the single-mode section. As the intensity is highest in the center of the beam, the carriers and thus the gain will be depleted in the center of the tapered section. This gives rise to higher amplification in the wings of the tapered section and the beam profile is changed so that the intensity on the output facet is close to uniform. Local heating in the diode laser will give rise to thermal effects such as lensing that changes the beam profile. In effect, the beam profile in the near and far field will be far from Gaussian, while in the focal region, the beam profile will be close to Gaussian with a strong central lobe and some low intensity side modes. This is illustrated in the example in Fig. 4 taken from a tapered diode laser at $1062 \mathrm{~nm}$ at $10 \mathrm{~W}$ output power. The non-Gaussian beam properties also increase the beam propagation ratio, $M^{2}$, significantly. Using the definition of the ISO11146 standard, the $\mathrm{M}^{2}$ value is often significantly larger than 1 . If one only considers the beam diameter at intensity levels larger than $1 / \mathrm{e}^{2}$, the measured $M^{2}$ value is often very close to 1 . In applications like nonlinear frequency conversion, the conversion is most efficient at high intensities and a combination of the $1 / \mathrm{e}^{2} M^{2}$ value and the power content of the central lobe of the beam is a good measure of the possible conversion efficiency in nonlinear frequency conversion. Unless stated otherwise, the $M^{2}$ values quoted in this paper follows the ISO11146 standard.

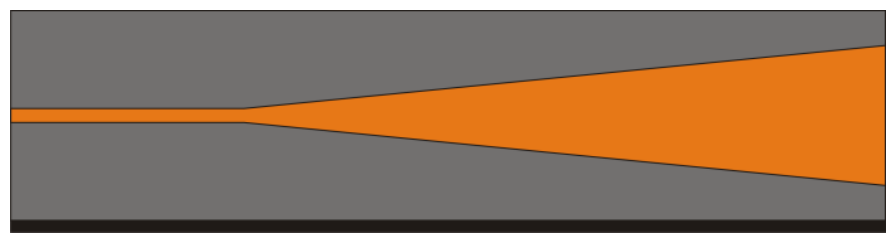

(a)

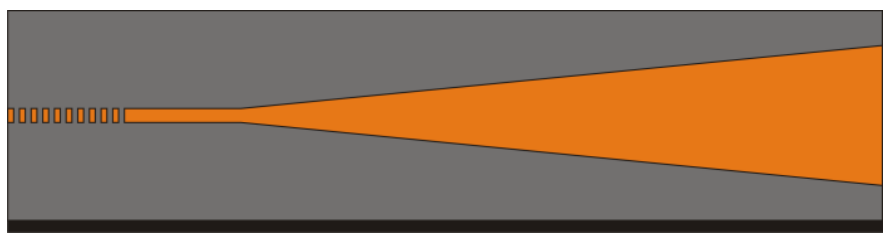

(b)

Fig. 3. Tapered diode laser structure (a) with a single mode waveguide emitting light into the tapered amplifier section. (b) Tapered diode laser with a build-in Bragg grating for wavelength selection.

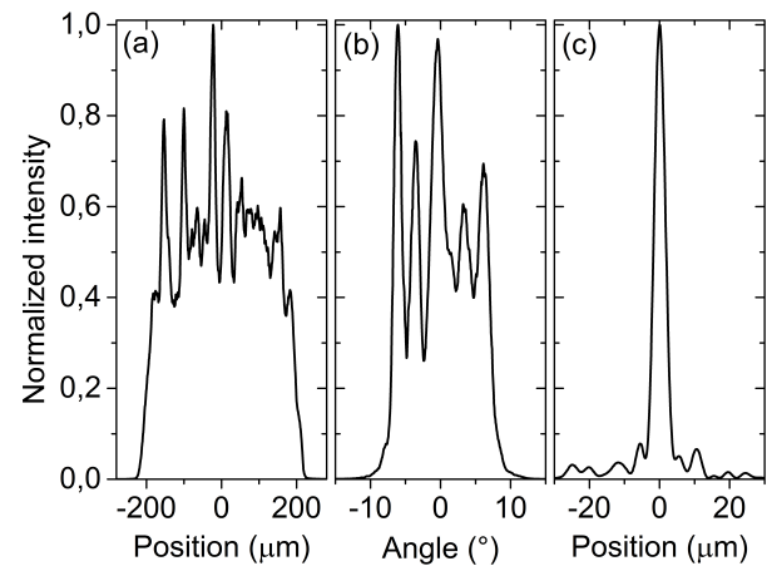

Fig. 4. Beam profile of a typical tapered diode laser in the near field (a), the far field (b) and the focus (c). The $M^{2}$ value for this particular laser is 8.7, whereas the $M^{2}$ value at the $1 / \mathrm{e}^{2}$ intensity level is 1.2 .

The spectral properties are also of high importance for frequency conversion as the nonlinear crystals have a limited wavelength acceptance bandwidth as illustrated in Fig. 2. The 
wavelength acceptance scales inversely with the length of the crystal [40]. In single-pass frequency conversion, longer crystals result in increased conversion efficiency but also reduced wavelength acceptance. Different methods exist for narrowing the spectral width of tapered diode lasers. These include the use of external cavities [19]-[23] and distributed Bragg reflector (DBR) or distributed feedback (DFB) gratings in the single-mode section of the lasers [25], [26], [67]-[69]. These methods routinely provide linewidths on the order of 1 $10 \mathrm{MHz}$, more than enough for efficient frequency conversion.

\section{POWER SCALING OF NONLINEAR FREQUENCY CONVERSION}

In this section, we provide examples of different laser systems based on single-pass nonlinear frequency conversion of tapered diode lasers. First we will give examples of lasers based on frequency doubling of single tapered diode lasers. Power scaling of the frequency doubling is possible using a cascade of nonlinear crystals. The power from a tapered diode laser is limited due to thermal effects. Combination of more tapered diode lasers and subsequent frequency conversion is a viable method for increasing the available output power. We provide examples of power scaling using sum frequency generation of beam combined tapered diode lasers.

\section{A. Second harmonic generation of tapered diode lasers} Second harmonic generation of tapered diode lasers was first demonstrated by Waarts et al. [70]. The output power of $3.7 \mathrm{~mW}$ was mainly limited by the $1 \mathrm{~W}$ output power from the tapered diode laser and by a decrease in beam quality at high power levels. The development of quasi phase matching gave access to significantly increased nonlinearities of corresponding periodically poled crystals and the output power levels achieved using single-pass SHG of tapered diode lasers have increased dramatically. Also, external cavity broad area diode laser systems have been demonstrated to be suitable for frequency doubling. Power levels of more than $100 \mathrm{~mW}$ has been achieved [71], [72]. Using either external cavity tapered diode lasers or master oscillator power amplifier configurations, up to $600 \mathrm{~mW}$ of light have been obtained in the blue spectral region with up to $4 \mathrm{~W}$ of fundamental input power [19], [73]-[75]. With the introduction of high power DBR tapered diode lasers, these power levels have been increased significantly in both the blue and green spectral region [27]-[29], [76]. Below we provide an example of the results obtained in the green spectral region.

A high power DBR tapered diode laser at $1062 \mathrm{~nm}$ was used [27], [77]. The laser was a $6 \mathrm{~mm}$ long device with a $1 \mathrm{~mm}$ long unpumped DBR grating, a $1 \mathrm{~mm}$ long single-mode ridge waveguide section and a $4 \mathrm{~mm}$ long tapered section with a $6^{\circ}$ taper angle as sketched in Fig. 3 (b). The ridge waveguide section and the tapered section were electrically separated in order to control output power, spatial and spectral behavior individually.

The DBR tapered diode laser was capable of emitting more than $9 \mathrm{~W}$ of output power at $14 \mathrm{~A}$ current to the tapered section and $300 \mathrm{~mA}$ to the ridge waveguide section as shown in Fig. 5 (a). The increase in power was linear with respect to the current and there was no sign of thermal roll-over even at the highest power level. The spectrum of the laser is governed by the DBR grating in the single-mode part of the laser. This DBR grating only reflects a very narrow spectral range and the laser will only operate at this specific wavelength. The result was a very narrow spectrum as shown in Fig. 5 (b) with a measured linewidth below $6 \mathrm{pm}$, limited by the resolution of the optical spectrum analyzer (Advantest Q8347). The wavelength of the laser can be changed by either changing the current to the laser or by operating at a different temperature. Typically, the wavelength changes by about $0.025 \mathrm{~nm}$ for a taper current change of $1 \mathrm{~A}$ and the wavelength change with laser temperature is about $0.08 \mathrm{~nm} / \mathrm{K}$.

SHG of the DBR tapered diode laser was carried out in a single pass through a periodically poled $\mathrm{MgO}$-doped $\mathrm{LiNbO}_{3}$ (PPMgLN) crystal. The experimental setup is shown in Fig. 6. The light from the DBR tapered diode laser was collimated in the fast axis by an aspherical lens with a focal length of $3.1 \mathrm{~mm}$. Due to astigmatism in the laser, the fast axis collimating lens focused the light in the slow axis and an additional cylindrical lens with $15 \mathrm{~mm}$ focal length was used to collimate the beam in the slow axis and correct for astigmatism. The beam was passed through an optical isolator with a half-wave plate on each side. Using the first half-wave plate it was possible to adjust the power passing the optical isolator without changing the operating conditions of the laser. The second half-wave plate was used to adjust the polarization for optimum SHG in the PPMgLN crystal. A $100 \mathrm{~mm}$ focal length spherical lens was used to focus the light to a beam waist radius of approximately $60 \mu \mathrm{m}$ in the PPMgLN crystal. This beam waist radius was significantly larger than optimum according to the theory of Boyd and Kleinman [44] but proved to give optimum conversion efficiency in the experiments. The deviation was caused by non-ideal beam properties of the tapered diode laser [78]. The PPMgLN crystal (HCPhotonics) was $30 \mathrm{~mm}$ long and poled with a period of $6.92 \mu \mathrm{m}$. The crystal was cut with an angle of $10^{\circ}$ and antireflection coated for $1064 \mathrm{~nm}$ and $532 \mathrm{~nm}$ on both end facets. The temperature of the crystal was stabilized at $35^{\circ} \mathrm{C}$ using an oven. After the PPMgLN crystal a filter was used to separate the green light from the infrared light.

The temperature of the laser was set at $14.3^{\circ} \mathrm{C}$ for optimum phase matching in the PPMgLN crystal with a wavelength of $1062.3 \mathrm{~nm}$. 8.52W of fundamental light was available before the crystal due to losses in the optical isolator and other optical components. From this input power, a maximum of $1.58 \mathrm{~W}$ of green light was generated and the power characteristics are shown in Fig. 7. The resulting power conversion efficiency was $18.5 \%$. The experimental results are fitted to the theory for SHG with depleted pump according to [41]

$$
P_{\mathrm{SHG}}=P_{\text {Laser }} \tanh ^{2}\left(\sqrt{\eta_{\text {SHG }} P_{\text {Laser }}}\right)
$$

Where $\eta_{S H G}$ is the nonlinear conversion efficiency of the PPMgLN crystal fitted to $2.5 \% / \mathrm{W}$. This nonlinear conversion efficiency is lower than $8 \% / \mathrm{W}$ achieved in [71], with the difference mainly caused by the beam quality of the fundamental laser. The beam quality of the generated green light was close to diffraction limited with $M^{2}<1.3$. This high beam quality compared to the output from the tapered diode laser is an outcome of the nonlinear frequency conversion process. The nonlinear conversion is most efficient at high pump power intensities and thus the central part of the focused 
beam from the tapered diode laser was more efficiently converted than the lower intensity side modes. As a result the generated green light had a nearly Gaussian beam profile.

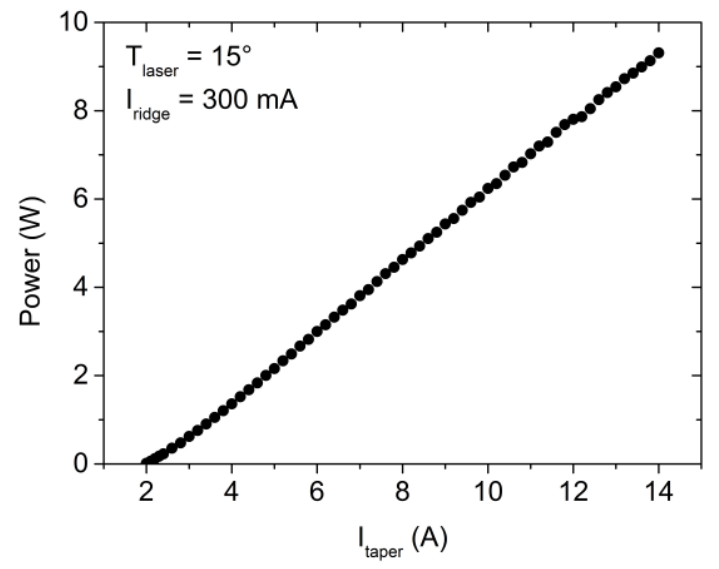

(a)

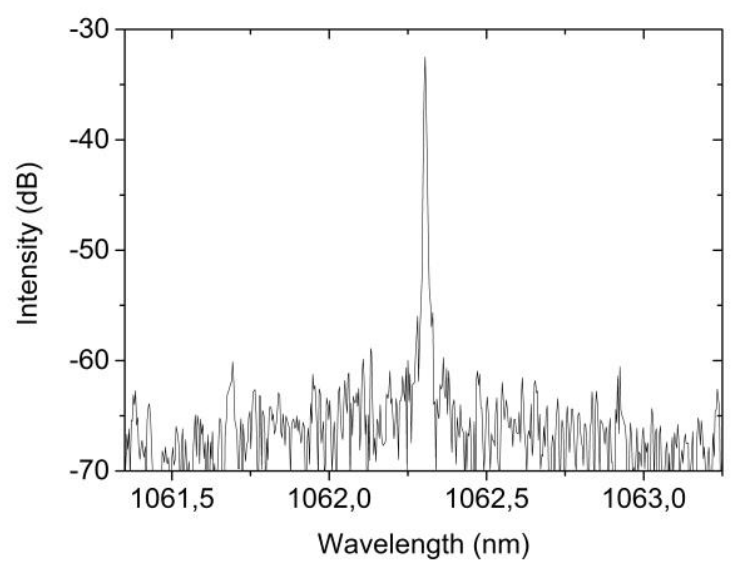

(b)

Fig. 5. Output power characteristics (a) and spectrum of the DBR tapered laser at $9 \mathrm{~W}$ output power (b).

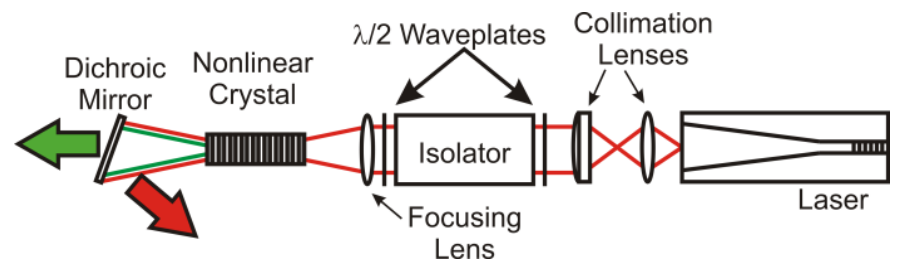

Fig. 6. Sketch of the experimental setup for single pass SHG of a DBR tapered diode laser.

Based on these results a compact prototype has been developed. The prototype had a foot print of $145 \times 82 \mathrm{~mm}$ and is shown in Fig. 8 together with a power stability test over 200 hours at $1.4 \mathrm{~W}$. The power was stabilized using feedback from a photodiode. The average power in the measurement was $1.407 \mathrm{~W}$ with maximum peak to peak deviations of $\pm 4 \mathrm{~mW}$.

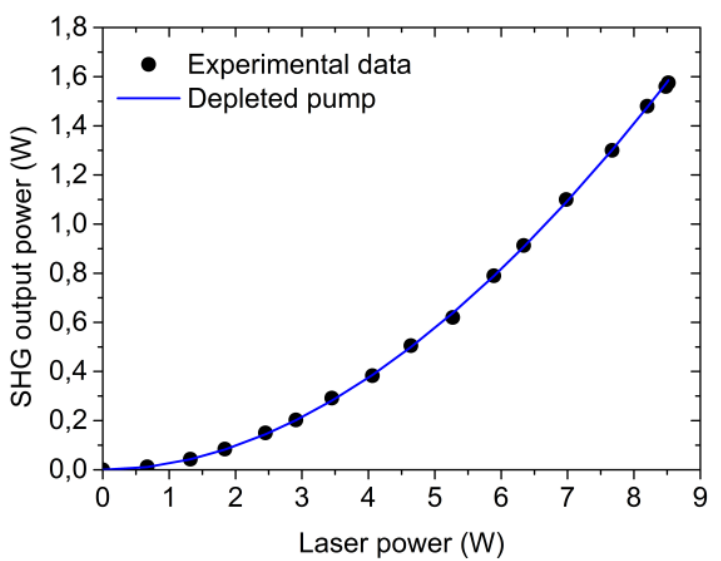

Fig. 7. SHG power vs. fundamental input power. The line is a numerical fit according to the depleted pump approximation (3) with a nonlinear conversion efficiency of $2.5 \% / \mathrm{W}$.

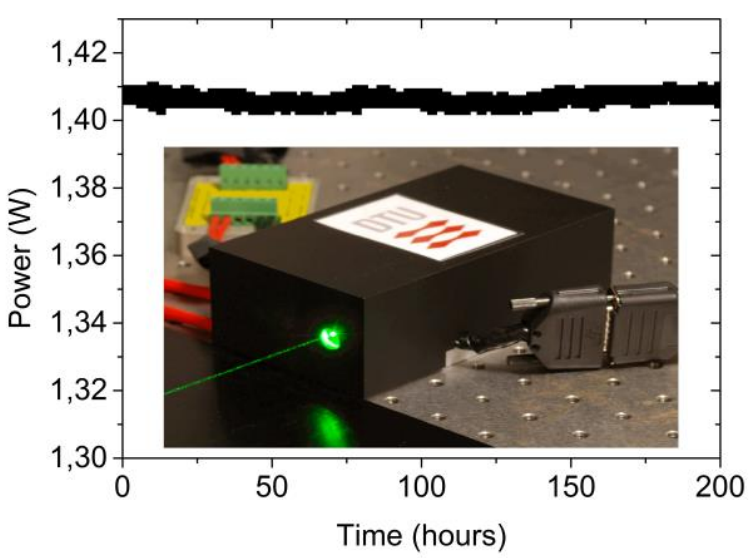

Fig. 8. Power stability measured over 200 hours for a compact prototype $1.4 \mathrm{~W}$ green laser based on SHG of a DBR tapered diode laser. The inset shows a picture of the compact prototype.

The versatility of diode lasers in terms of emission wavelength enables generation of light at other wavelengths. High power DBR tapered diode lasers have been demonstrated at wavelengths ranging from $920 \mathrm{~nm}$ to $1120 \mathrm{~nm}$ [77], [79], [80]. This provides access to visible light in the $460-560 \mathrm{~nm}$ wavelength range and possibly this range may soon be extended to $580 \mathrm{~nm}$ to include the wavelength of maximum absorption in hemoglobin.

\section{B. Cascaded frequency doubling}

A further increase in output power using single-pass frequency doubling in a single nonlinear crystal has not been possible as the beam properties of the tapered diode laser decrease at higher output power and the nonlinear frequency conversion efficiency decreases. Two possible routes to increasing the second harmonic output power is to use either multiple passes in the same nonlinear crystal [81], [82] or cascaded frequency doubling in a series of crystals [83]-[86]. Cascaded frequency doubling in two identical crystals could in principle result in a four-fold increase in generated power following the relation

$$
P_{\mathrm{SHG}}=\eta_{S H G} N^{2} P_{\text {Laser }}^{2}
$$


Where $\mathrm{N}$ is the number of crystals in the cascade [83]. In practice, however, lower conversion efficiencies are normally obtained due to depletion of the input fundamental light, nonperfect beam properties and phase compensation and risk of damage to nonlinear crystals with optimum focusing [85].

Cascaded SHG of a DBR tapered diode laser in two PPMgLN crystals was recently demonstrated in a setup as shown in Fig. 9 [87]. The setup was very similar to Fig. 6 with the addition of a second nonlinear crystal. Two curved mirrors were used to achromatically re-image both the fundamental and second harmonic light exiting the first nonlinear crystal into the second nonlinear crystal. In order for the second harmonic light generated in the second crystal to interfere constructively with the light generated in the first crystal, it was important to ensure that their relative phases are correct. In the setup in Fig. 9 , phase compensation was achieved using the dispersion of a plane glass plate. By rotating the plate, the proper phase relation between the fundamental and second harmonic beams can be realized at the input face of the second crystal.

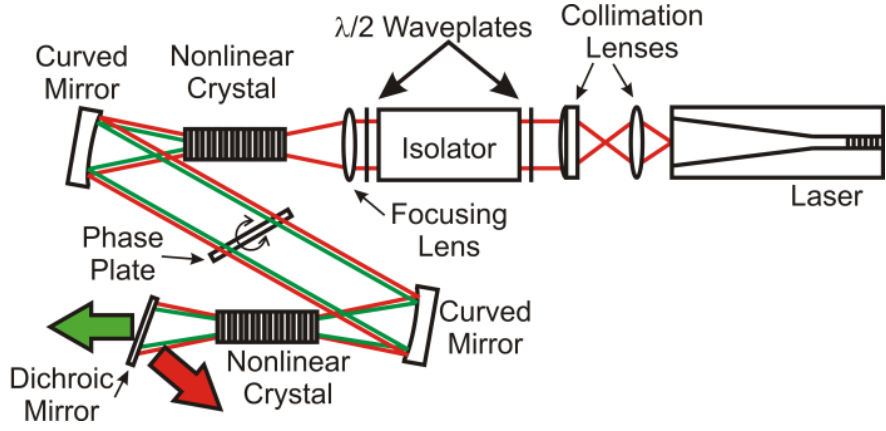

Fig. 9. Sketch of the experimental setup for cascaded SHG of a DBR tapered diode laser.

First experiments on cascaded frequency doubling of a DBR tapered diode laser in two $30 \mathrm{~mm}$ long PPMgLN crystals have generated $3.5 \mathrm{~W}$ of green light with an optical-optical conversion efficiency of $35 \%$. Possibly, more second harmonic power can be generated by addition of more cascaded crystals. Ultimately, the output power is limited by the power from the tapered diode laser.

The limitation on the frequency doubled output power caused by beam quality degradation of the tapered diode lasers at increased output power necessitates considering alternative means of further increasing the output power. The output power of the fundamental light can be increased by combining the beams from multiple tapered diode lasers. Frequency conversion of this combined beam will lead to higher output powers in the frequency converted beam.

\section{Spectral beam combining and sum frequency} generation between two tapered diode lasers

Sum frequency generation is a method used to generate light at the sum frequency of two interacting fundamental beams with different frequencies. It is often used to generate light at wavelengths that are not easily accessible by other means. One well-known example is generation of light at the sodium D2 resonance wavelength at $589 \mathrm{~nm}$ by SFG between two $\mathrm{Nd}$ :YAG lasers at $1064 \mathrm{~nm}$ and $1319 \mathrm{~nm}$ [88]-[90]. Other examples include the generation of blue light at $460 \mathrm{~nm}$ by mixing $808 \mathrm{~nm}$ diode lasers with lasers at $1064 \mathrm{~nm}$ [91]-[94].
These examples used lasers widely separated in wavelength and these can easily be combined using dichroic mirrors. Similar DBR tapered diode lasers around $1062 \mathrm{~nm}$ cannot be separated in wavelength by more than a few nanometers and dichroic coatings cannot be used to combine these lasers. Polarization combining [95] could be used but as periodically poled crystals are desirable, highest possible nonlinear efficiency requires the polarization of the two interacting beams to be parallel. Spectral beam combining using Bragg gratings is a way of combining lasers at closely spaced wavelengths [96]-[98]. This method is often used to increase the power from fiber laser based systems while keeping the power in each fiber laser relatively low. Here, the same technique was used to keep the power level from the tapered diode lasers at a reasonable level, while achieving higher power in the combined beam. One unique feature regarding this method applied to diode lasers is that it enables combining two identical lasers just by performing a slight detuning of the wavelengths. Adapting the crystal to the selected wavelengths ensures efficient frequency conversion. The beam properties of the combined beam are a combination of the beam properties of the individual lasers. Hence combining two DBR tapered diode lasers with good beam quality will result in a combined beam with good beam quality suitable for SFG. By properly selecting the wavelengths of the interacting lasers, it is possible to generate the optimum wavelength for the application in mind. Below we provide an example of generation of green light by SFG of two spectrally combined DBR tapered lasers [30].

The experimental setup is shown schematically in Fig. 10. It consisted of two DBR tapered diode lasers as shown in Fig. 3 (b) with performance similar to the one shown in Fig. 5. The lasers were both collimated in the fast and slow axes using pairs of aspherical lenses with $3.1 \mathrm{~mm}$ focal length and cylindrical lenses with $15 \mathrm{~mm}$ focal length. A reflecting volume Bragg grating was used for spectral beam combining. The grating had an average diffraction efficiency higher than $99 \%$ with a spectral acceptance bandwidth of $0.3 \mathrm{~nm}$ (FWHM). The grating dimensions were $10 \times 10 \times 3.4 \mathrm{~mm}^{3}$ $(\mathrm{W} \times \mathrm{H} \times \mathrm{L})$. Efficient spectral beam combining was achieved by optimizing the incident angle on the grating and the wavelengths of the two lasers. Laser 1 was tuned to a wavelength outside the spectral acceptance bandwidth and was transmitted through the grating while laser 2 was diffracted and in this way combined with the beam from laser 1 . Efficient spectral beam combination was realized by the simple tunability of the wavelength of the DBR tapered diode lasers by temperature. A $30 \mathrm{~dB}$ optical isolator was inserted to prevent feedback to the diode lasers. Two half-wave plates were inserted to rotate the polarization for optimum efficiency in the nonlinear crystal and to enable power adjustment without changing the beam parameters. An achromatic lens with $75 \mathrm{~mm}$ focal length focused the light to a beam waist radius of approximately $42 \mu \mathrm{m}$ inside the $30 \mathrm{~mm}$ long PPMgLN crystal. The crystal was poled with a period of $6.92 \mu \mathrm{m}$ and anti-reflection coated at both end facets. The crystal was kept in a temperature controlled oven to enable temperature phase matching. A dichroic mirror separated the residual fundamental light from the generated green light. 


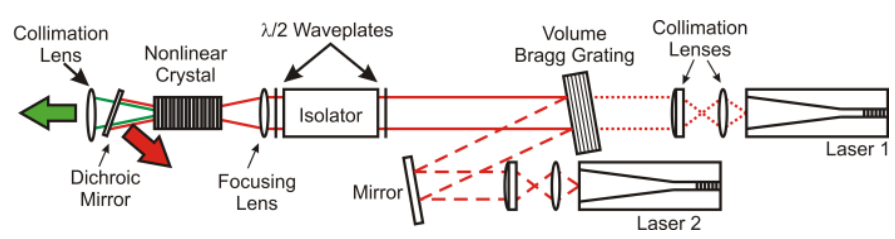

Fig. 10. Experimental setup for sum frequency generation of two spectrally combined DBR tapered diode lasers.

The tapered diode lasers were operated under similar conditions with $300 \mathrm{~mA}$ current to the ridge sections and $16 \mathrm{~A}$ current to the tapered sections. The temperatures of the two lasers were $23.1^{\circ} \mathrm{C}$ for laser 1 and $15.9^{\circ} \mathrm{C}$ for laser 2 to ensure proper wavelength separation for beam combination. The wavelength of laser 1 was $1063.3 \mathrm{~nm}$ while it was $1062.3 \mathrm{~nm}$ for laser 2 . The combining efficiency of the lasers was higher than $90 \%$ and the beam propagation factors were $M^{2}=2$ in the fast axis and $M^{2}=5.1$ in the slow axis.

At a combined input power of $15.7 \mathrm{~W}$ into the PPMgLN crystal, up to $3.9 \mathrm{~W}$ of green light was generated as shown in Fig. 11 (a). This corresponds to an optical-optical conversion efficiency of $24.8 \%$ and a nonlinear conversion efficiency of $2.6 \% / \mathrm{W}$. This power level was higher than the sum of SHG of the individual lasers. SHG of the two lasers generated $1.5-$ $1.6 \mathrm{~W}$ of green light and thus the improvement by using SFG was a factor of 2.5 over SHG of a single laser. The level of improvement could in principle be as large as a factor of 4 assuming the non-depleted pump approximation and identical lasers. The deviation in the experiments could to a large extent be explained by pump depletion but imperfect overlap and reduced beam quality also have significant impact on the conversion efficiency.

The spectral properties of the generated light were governed by the spectral properties of the two interacting lasers. As the two DBR tapered diode lasers were both narrowband, the generated green light also had a narrow linewidth of less than $5 \mathrm{pm}$ limited by the resolution of the optical spectrum analyzer. The generated green light had close to diffractionlimited beam quality with $M^{2}<1.1$ in the fast axis and $M^{2}<1.3$ in the slow axis as shown in Fig. 12. The large improvement compared to the beam propagation factors of the spectrally combined beam was related to nonlinear beam clean-up [99]. Here it was shown that the beam profile of the generated beam is highly dependent on the overlap of the two fundamental beams. The good beam quality also indicates the absence of power handling problems in the PPMgLN crystal. This improvement in performance by combining two DBR tapered diode lasers over SHG of a single laser holds promise for more compact and efficient visible laser systems in the multiple watt range in the near future and shows that it is possible to efficiently generate coherent light by SFG between two mutually incoherent fundamental lasers. However, the generated power is still limited by the performance of the two lasers. Spectral beam combination is not limited to two lasers but can be expanded almost indefinitely.

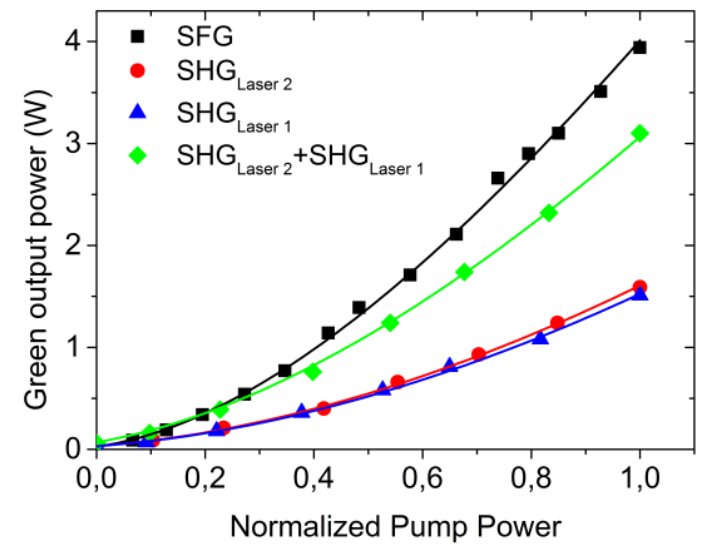

(a)

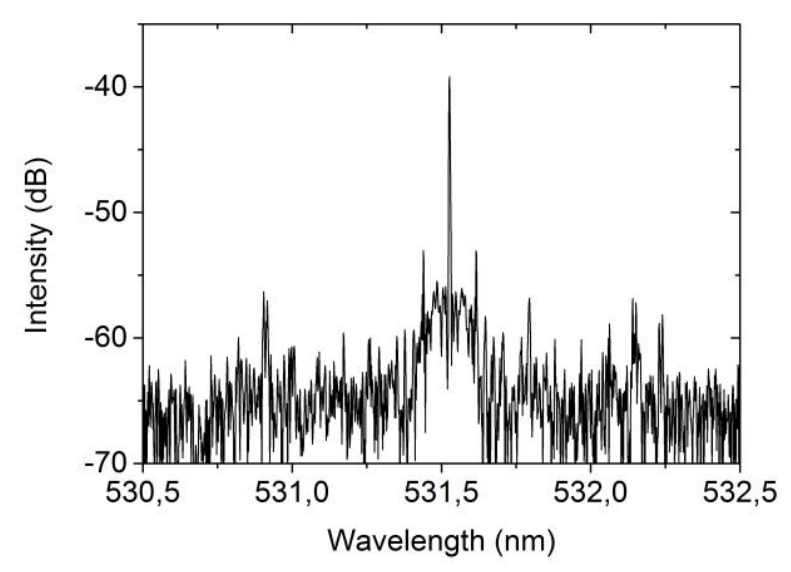

(b)

Fig. 11. Generated power by SFG of two spectrally combined DBR tapered diode lasers (a). The pump power is normalized to the maximum available pump power for the individual processes. Spectrum of the generated green light (b).

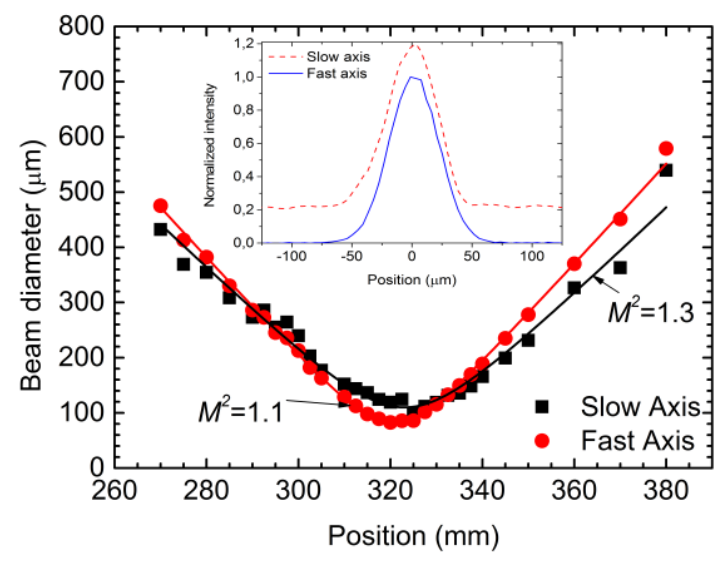

Fig. 12. Measured beam propagation parameters for the fast and slow axis of the generated green light. The inset shows focus beam profiles in the fast and slow axis. 


\section{Spectral beam combination of three lasers and simultaneous $S H G$ and $S F G$}

Spectral beam combination of fiber lasers have been demonstrated with up to 5 lasers [97] and with diode lasers significantly more lasers have been combined [100]-[104]. If the wavelengths of these combined lasers are chosen properly, it is possible to obtain efficient frequency conversion of several lasers simultaneously and generation of light at a single wavelength can be achieved while operating the nonlinear crystal at a constant temperature. In the case described below, three lasers are spectrally combined with a multiplexed volume Bragg grating (VBG). The wavelengths of the lasers were chosen so as to perform efficient SHG of the laser with the center wavelength (laser 2), while the wavelengths of the other two lasers (laser 1 and 3) were positioned on both sides of the center wavelength and tuned for efficient SFG. It is also possible to combine four lasers and simultaneously perform SFG between pairs of lasers and adding more lasers and performing either SHG or SFG will be possible as long as phase matching can be obtained in the nonlinear crystal. If the generated wavelength is fixed at 532 $\mathrm{nm}$ and the fundamental wavelengths are selected to generate this wavelength, an acceptance bandwidth (FWHM) of 23.8 $\mathrm{nm}$ can be calculated for a $30 \mathrm{~mm}$ long PPMgLN crystal as shown in Fig. 13. If the fundamental wavelengths are separated by approximately $0.5 \mathrm{~nm}$, up to 23 pairs of fundamental beams can efficiently generate light at the same wavelength.

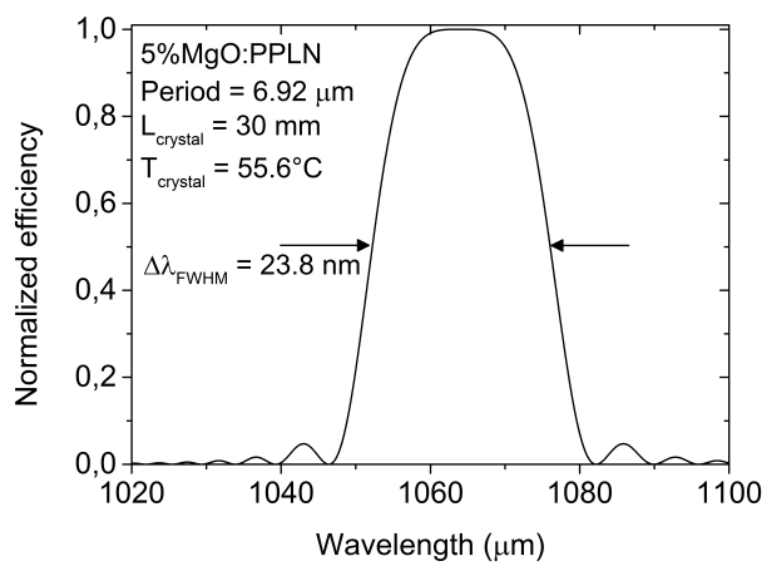

Fig. 13. Simulated acceptance bandwidth for SFG into a fixed wavelength of $532 \mathrm{~nm}$ in a $30 \mathrm{~mm}$ long PPMgLN crystal with a poling period of $6.92 \mu \mathrm{m}$ at a temperature of $55.6^{\circ} \mathrm{C}$.

For the first time, SFG and SHG of three independent lasers were performed realizing one combined output beam. A sketch of the experimental configuration for the combination of three DBR tapered lasers and simultaneous SHG and SFG of these lasers is shown in Fig. 14. The setup was similar to the one depicted in Fig. 10 but with the addition of a laser and the use of a multiplexed VBG. Laser 2 was frequency doubled in the nonlinear crystal and SFG was obtained between lasers 1 and 3 . The use of a multiplexed VBG set a relation between the wavelengths of lasers 2 and 3 in order for them to be combined spatially. The wavelength of laser 1 was still freely chosen as long as it was outside the diffraction bands of the
VBG. The reflecting VBG $\left(10 \times 10 \times 3.99 \mathrm{~mm}^{3}\right)$ had two diffraction wavelengths separated by $0.5 \mathrm{~nm}$ with an input angle difference of $20^{\circ}$ and an output angle of $1^{\circ}$ relative to the VBG normal. The two individual gratings had an average diffraction efficiency higher than $99 \%$ and acceptance bandwidths of below $0.3 \mathrm{~nm}$.

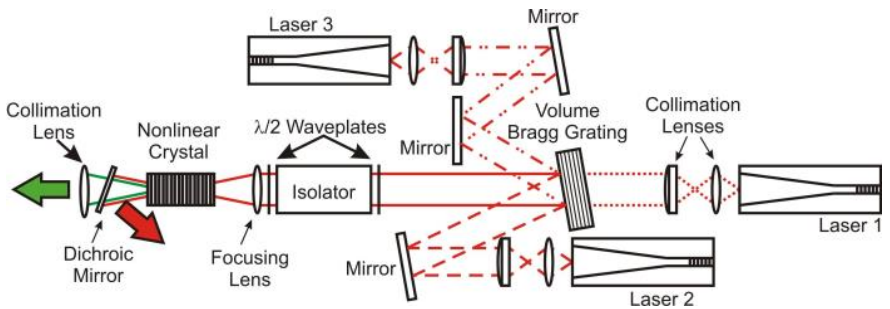

Fig. 14. Experimental setup for spectral beam combining of three DBR tapered lasers in a multiplexed VBG and subsequent SHG of laser 2 and SFG between laser 1 and laser 3 .

In this first demonstration of the principle, the lasers were operated at relatively low power with a current of $300 \mathrm{~mA}$ to the ridge section and $4 \mathrm{~A}$ to the tapered section of the lasers. This provided an output power of about $1 \mathrm{~W}$ for each laser. Due to the VBG, the wavelengths of laser 2 and 3 were fixed at $1063.19 \mathrm{~nm}$ and $1062.05 \mathrm{~nm}$, respectively. The SHG of laser 2 lead to a wavelength of $531.59 \mathrm{~nm}$ and this meant that laser 1 must emit at $1064.31 \mathrm{~nm}$. This dictated the laser temperature to be $39.1{ }^{\circ} \mathrm{C}$ and this high temperature lowered the emitted power to about $630 \mathrm{~mW}$. The pump power for SHG was $1.03 \mathrm{~W}$ and resulted in $40 \mathrm{~mW}$ of power in the generated green beam. SFG between lasers 1 and 3 and their combined $1.76 \mathrm{~W}$ output power resulted in $100 \mathrm{~mW}$ of light in the generated beam. When both processes were performed simultaneously, the output power sums to generate $140 \mathrm{~mW}$ in the green output beam. Increasing the output power to the maximum possible from the DBR tapered lasers could potentially result in the sum of the powers obtainable with SFG and SHG, i.e. about $5.5 \mathrm{~W}$ referring to the results described in the previous sections.

By tuning the wavelength of laser 1 , it was possible to generate the same wavelength by both SHG and SFG. This had the effect of causing interference effects similar to superposition of coherent waves [105]. These interference effects caused random power fluctuations not seen in the individual contributions from SHG and SFG. If the emission wavelength of laser 1 was slightly detuned, these instabilities disappeared. For laser 1 tuned to generate the same wavelength as SHG of laser 2, the spectrum of the combined SHG and SFG output beam is shown in Fig. 15. The line width was narrower than $5 \mathrm{pm}$ and the side mode suppression was about $10 \mathrm{~dB}$. This relatively low side mode suppression was also seen in the infrared beam and may be caused by the DBR grating or undesirable feedback effects. The beam propagation factor $M^{2}$ of the combined green output beam was 1.6 in the fast axis and 2.3 in the slow axis. This unexpectedly high $M^{2}$ factor is expected to be improved by better overlap of the fundamental laser beams. SHG and SFG between three combined beams enabled a total of six nonlinear interactions. By changing the PPMgLN temperature it was possible to clearly distinguish the different interactions as seen in Fig. 16. 
The only exception was the central peak where SFG of lasers 1 and 3 and SHG of laser 2 overlap.

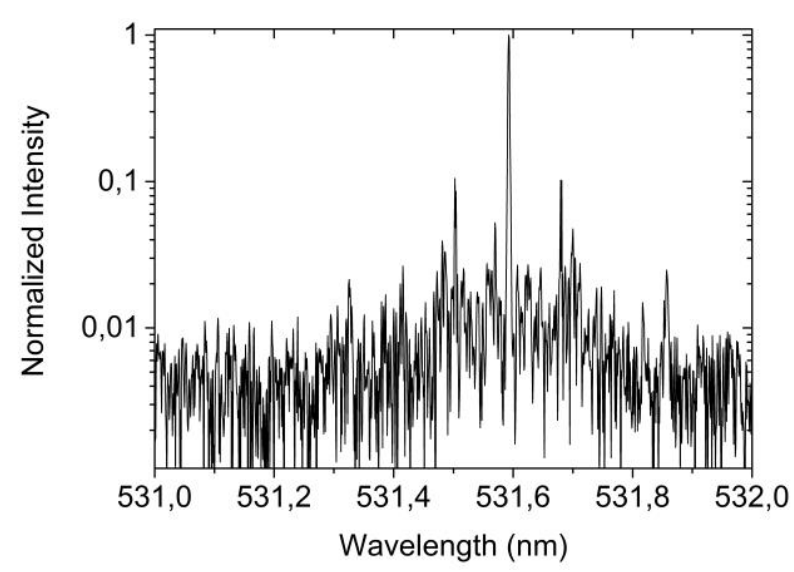

Fig. 15. Spectrum of the green output beam, when the lasers were tuned to provide the same green wavelength.

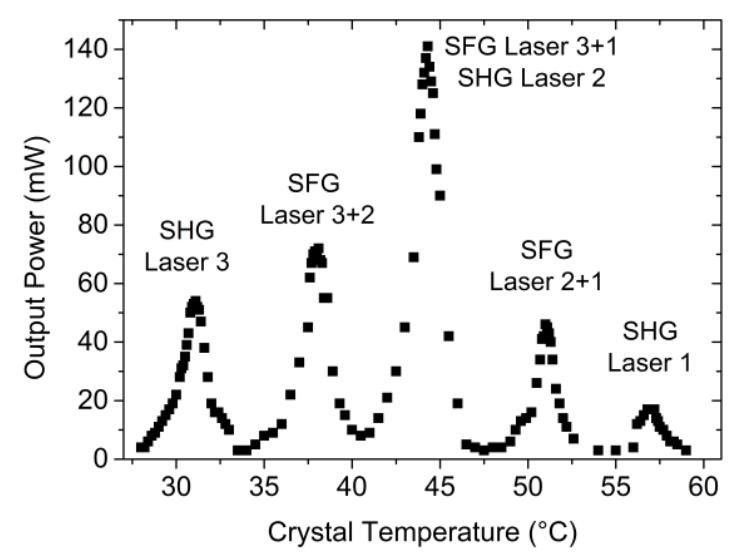

Fig. 16. Temperature phase matching measurements with three interacting lasers.

\section{TI:SAPPHIRE LASERS PUMPED BY FREQUENCY DOUBLED TAPERED DIODE LASERS}

A commonly used laser system within biophotonics diagnostics and imaging applications is the mode-locked Ti:sapphire laser, due to their ultrashort pulses with a correspondingly large bandwidth. Ti:sapphire lasers have their main absorption band in the blue-green spectral range and have been pumped using various lasers in this spectral range [31], [106], [107]. The pump power required for pumping Ti:sapphire lasers depends strongly on the intended application of the Ti:sapphire laser. High output power from the Ti:sapphire laser requires high pump power but for most biophotonics applications, only very limited power is allowed for laser safety reasons. Furthermore, the bandwidth of the Ti:sapphire laser increases with pump power, so in order to achieve high resolution imaging, a high pump power is required. However, new developments of low loss resonator designs have allowed for pump power levels of less than $2 \mathrm{~W}$ providing high bandwidth Ti:sapphire lasers [31], [108], [109]. Currently, the most commonly used pump lasers for Ti:sapphire lasers are frequency doubled diode-pumped solid state (DPSS) lasers. These lasers typically provide high output power beams with excellent beam properties at a wavelength of $532 \mathrm{~nm}$. The main drawbacks of DPSS lasers are their high cost, large footprint and potentially less favorable noise properties in the $\mathrm{kHz}$ frequency range [110]. An approach to more cost effective and compact pump lasers would be to use the direct emission of diode lasers. One further advantage of using the direct output of diode lasers is that the typical relaxation oscillation frequency of diode lasers is in the $\mathrm{GHz}$ range and noise originating from relaxation oscillations will be filtered by the long excited state lifetime in Ti:sapphire crystals. Pumping with blue emitting diode lasers has been demonstrated recently [111], [112] and mode-locking of the Ti:sapphire laser has also been demonstrated [113], [114]. High power blue diode lasers are broad area lasers giving reduced beam quality in the slow axis of the laser, which might reduce the pump efficiency and also limits the modelocking capabilities. Furthermore, degradation effects within the Ti:sapphire crystals have been observed when pumping Ti:sapphire lasers with blue diode lasers. The frequency doubled diode lasers described in the previous sections constitute a possibly better suited pump source for Ti:sapphire lasers. A frequency converted diode laser system removes the need for a high finesse laser resonator enabling compact laser systems with low requirements on positioning of components. This facilitates cheap mass production. In the paragraphs below we provide results of a Ti:sapphire laser directly pumped by a frequency doubled diode laser [31].

A frequency doubled DBR tapered diode laser similar to the one depicted in Fig. 8 but with slightly different specifications was used as pump source for a Ti:sapphire laser in a setup described schematically in Fig. 17. The maximum output power of the pump laser was $1.3 \mathrm{~W}$ at $531.2 \mathrm{~nm}$ with a beam propagation ratio $M^{2}$ of 1.3 and 1.4 along the fast and slow axes, respectively. The beam was collimated to a beam diameter of approximately $2 \mathrm{~mm}$ and focused into the Ti:sapphire crystal using a $35 \mathrm{~mm}$ focal length spherical lens. The Ti:sapphire crystal positioned at Brewster angle was $3 \mathrm{~mm}$ long and had a figure of merit $>150$ and an absorption coefficient of $4.5 \mathrm{~cm}^{-1}$. The laser oscillator was an X-folded cavity with several extra folds to minimize the footprint and several mirrors in the cavity are dispersion compensating [108]. Two curved mirrors with $50 \mathrm{~mm}$ radius of curvature surrounded the Ti:sapphire crystal and the cavity lasing mode exhibited an $18 \mu \mathrm{m}$ beam waist inside the crystal. A mirror with 3\% transmission was used for output coupling. The total cavity length was about $1.75 \mathrm{~m}$ yielding a repetition rate of approximately $80 \mathrm{MHz}$. One end mirror was positioned on a stage for initiation of the Kerr-lens mode-locking. 


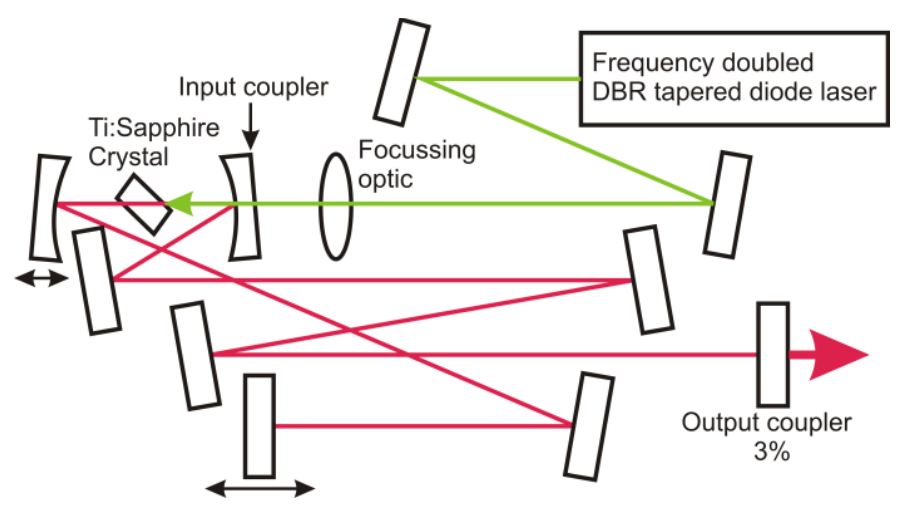

Fig. 17. Schematic layout of the $\mathrm{CW}$ or mode-locked Ti:sapphire laser oscillator pumped by the frequency doubled DBR tapered diode laser.

In CW operation, the Ti:sapphire laser was capable of delivering $110 \mathrm{~mW}$ of output power as shown in Fig. 18. Kerrlens mode-locking was initiated by shifting the stability of the cavity by moving one curved mirror and by inducing a perturbation on the movable end mirror. Under mode-locked operation, up to $82 \mathrm{~mW}$ output power was obtained. The generated mode-locked pulses had a spectral width of approximately $115 \mathrm{~nm}$ (FWHM) and an autocorrelator measurement of the pulses revealed a pulse duration of less than $20 \mathrm{fs}$ as shown in Fig. 19. The peak in the spectrum at $700 \mathrm{~nm}$ was caused by a sharp phase change in the chirped mirrors.

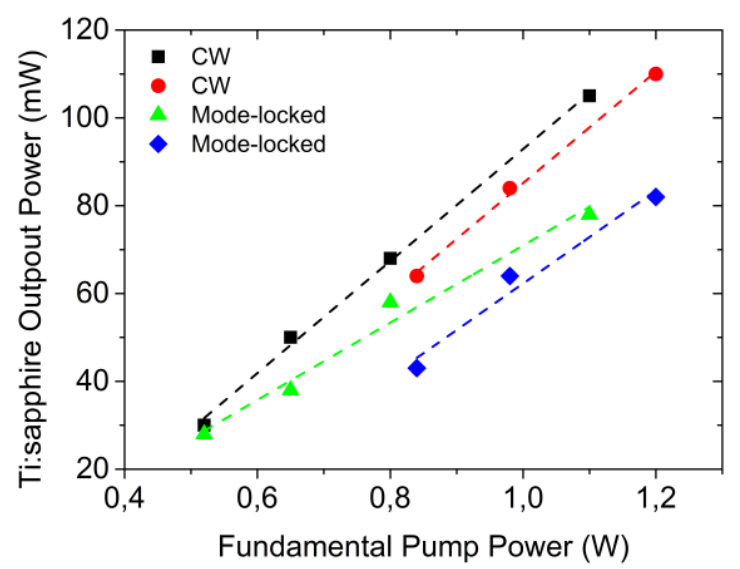

Fig. 18. Output power from the Ti:sapphire laser pumped by a frequency doubled DBR tapered diode laser with two differently collimated input beams in $\mathrm{CW}$ and mode-locked operation.

By these results it was shown that frequency doubled DBR tapered diode lasers are suitable for pumping ultrafast modelocked Ti:sapphire lasers. A comparison with a commercial DPSS laser revealed small differences in pumping performance in favor of the DPSS laser. However, this may be explained by the slightly better beam quality of the DPSS laser and that the Ti:sapphire laser cavity was optimized for the DPSS laser beam. Nevertheless, the overall efficiency was improved by more than a factor of 2 by using the frequency doubled DBR tapered diode laser due to the higher electrooptical efficiency.

This demonstration opens a new range of application possibilities using the Ti:sapphire laser pumped by the frequency doubled DBR tapered diode laser. Within optical imaging in biophotonics, Ti:sapphire lasers are widely used and in the next section, we discuss optical coherence tomography as an application example.

\section{OPTICAL COHERENCE TOMOGRAPHY}

Optical coherence tomography is a well-established imaging technique enabling depth resolved images with high resolution [6], [7]. The resolution is inversely proportional to the bandwidth of the light source and as such the wide bandwidth of a mode-locked Ti:sapphire laser makes it an ideal source. The main limitation of using Ti:sapphire lasers in OCT systems is the relatively high cost of such lasers, in turn mainly determined by the DPSS pump sources used. A shift of pump sources to frequency doubled DBR tapered diode lasers may provide access to lower cost and more compact Ti:sapphire lasers in the future. Here we will show initial results of in vivo OCT imaging of the retina and skin using a Ti:sapphire laser pumped by a frequency doubled DBR tapered diode laser [109].

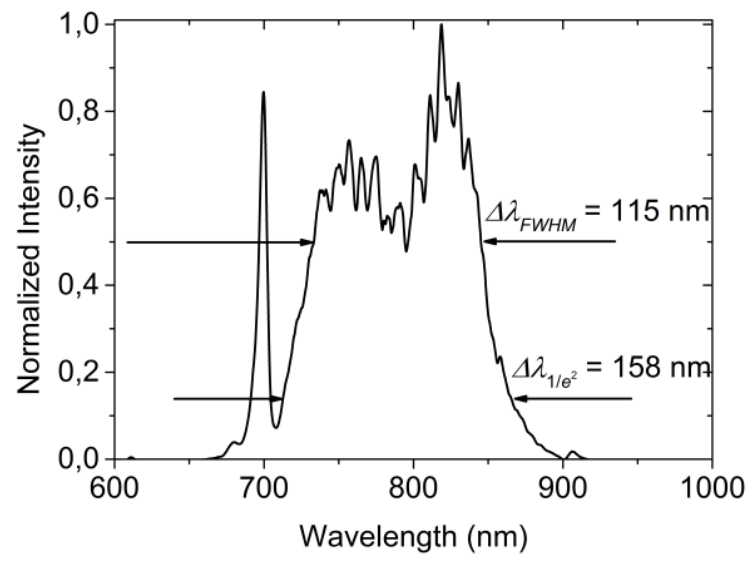

(a)

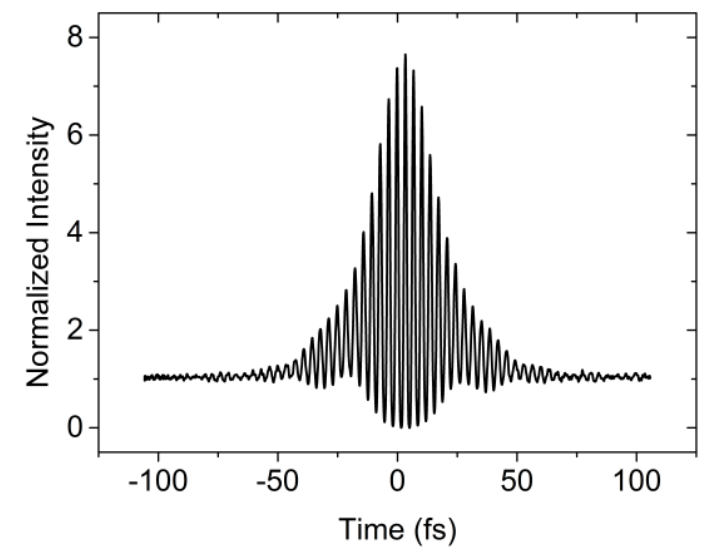

(b)

Fig. 19. Spectrum of the output pulses (a) with a bandwidth of $115 \mathrm{~nm}$ and autocorrelation trace (b) showing less than $20 \mathrm{fs}$ pulse duration. 


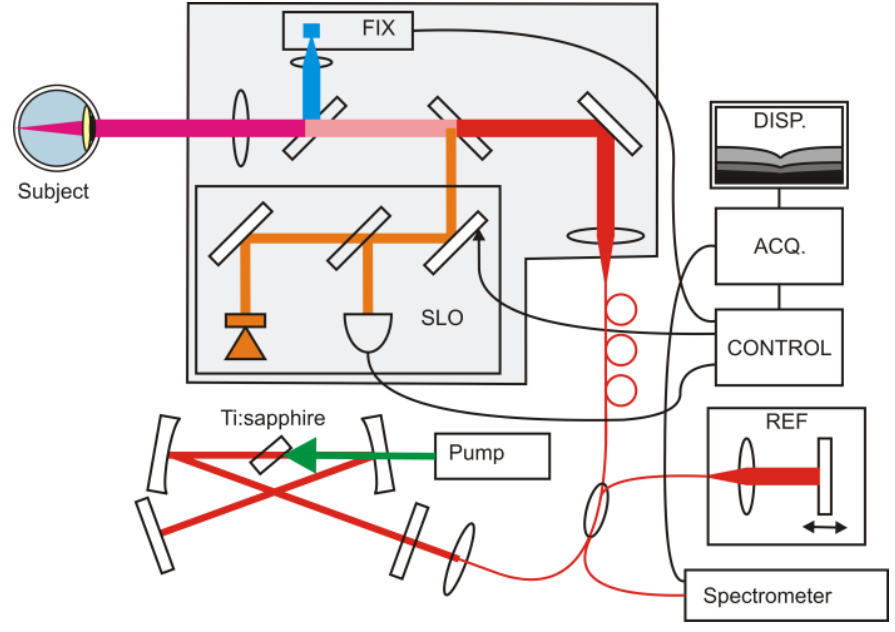

Fig. 20. Eye-tracked OCT system operating at $800 \mathrm{~nm}$.

The OCT system was a modified Spectralis OCT device (Heidelberg-Engineering) with built-in fixation target and eyetracking device. The OCT system was modified to enable use of wide bandwidth light sources at $800 \mathrm{~nm}$ and $1060 \mathrm{~nm}$. The entire system is shown schematically in Fig. 20.

A blue LED was used as fixation light source and an integrated confocal scanning laser ophthalmoscope (SLO) corrects for eye-motion. The light from the Ti:sapphire laser was coupled to an optical fiber and the output power from the fiber was regulated to give a maximum of $0.8 \mathrm{~mW}$ at the cornea well below the safety limits. A scanning reference arm provided the depth information and a grating based spectrometer was used for monitoring the laser spectrum. Control hardware regulated the fixation light, scanning laser ophthalmoscope and eye-motion correction, respectively. A camera link acquisition was processed in real time and displayed for viewing.

The Ti:sapphire laser was modified to provide stable modelocked operation with an output power of $80 \mathrm{~mW}$ at $1.4 \mathrm{~W}$ pump power. The spectrum of the Ti:sapphire laser is shown in Fig. 21 with a spectral width of $90 \mathrm{~nm}$ (FWHM) and $>200 \mathrm{~nm}$ full width. This provides an axial resolution of sub $4 \mu \mathrm{m}$. This resolution is sufficient for high resolution OCT imaging of the skin in vivo. Fig. 22 shows a tomogram of the left index finger of a healthy subject with a penetration depth of about $800 \mu \mathrm{m}$ clearly visualizing subsurface structures of normal skin, including the epidermis, dermoepidermal junction, dermis and sweat ducts.

Tomograms of the retina (a) and the optic nerve head (b) of a healthy subject are shown in Fig. 23. The use of a broad bandwidth laser source enabled despeckling at high axial resolution and allows visualization of detailed retinal anatomy including all main retinal layers such as retinal nerve fiber layer, ganglion cell layer, inner nuclear layer, outer nuclear layer, external limiting membrane, inner/outer segment, the separation of the RPE-Choriocapillaris complex fine structure or the choroid and the optic nerve fine structure such as the lamina cribrosa where the identification of structural changes are crucial in the diagnosis of glaucoma.

The high axial resolution and contrast in the OCT images clearly illustrate the potential of using Ti:sapphire lasers pumped by frequency doubled DBR tapered diode lasers for biophotonic imaging. The lower cost and footprint of these pump lasers should enable significant widespread adoption in biophotonic diagnostics.

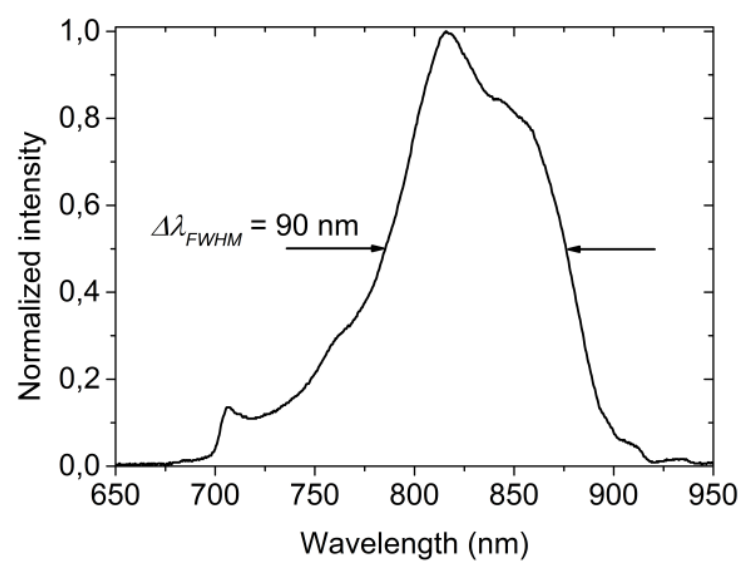

Fig. 21. Spectrum of the Ti:sapphire laser used for OCT measurements. The spectral width is $90 \mathrm{~nm}$ (FWHM).

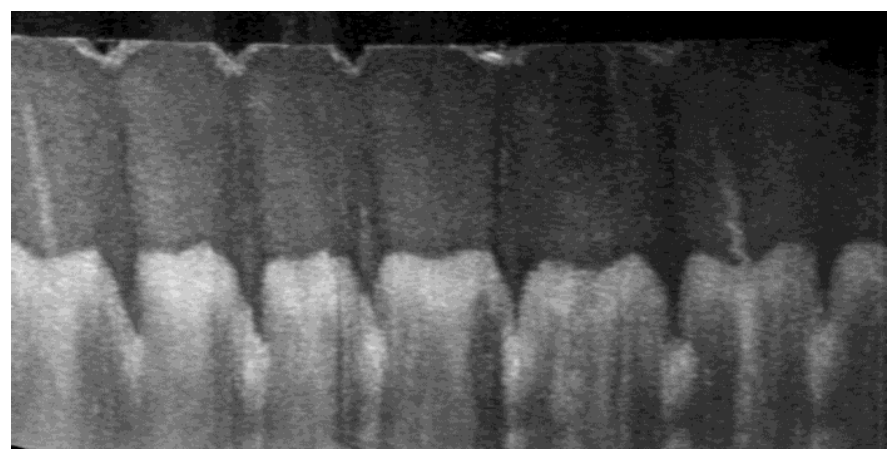

Fig. 22. OCT images of skin using the Ti:sapphire laser pumped by a frequency doubled DBR tapered diode laser.

\section{SUMMARY}

Frequency converted tapered diode lasers show great potential for many applications within biophotonics. The demonstration of several watts of output power in the green spectral range from purely diode laser based systems illustrates the application potential of these lasers. Of particular interest is direct pumping of mode-locked Ti:sapphire lasers to generate ultrashort pulses from highly compact and cost-effective laser system. These laser systems should enable further widespread adoption of biophotonics imaging like optical coherence tomography and multi-photon imaging. Power scaling of the green pump lasers will enable larger bandwidth Ti:sapphire lasers and e.g. increase resolution in optical coherence tomography.

Expansion of the wavelength range into the yellow spectral region opens for applications within photocoagulation in ophthalmology and treatment of vascular lesions. In these applications, a higher power level will enable faster and more precise treatments. 


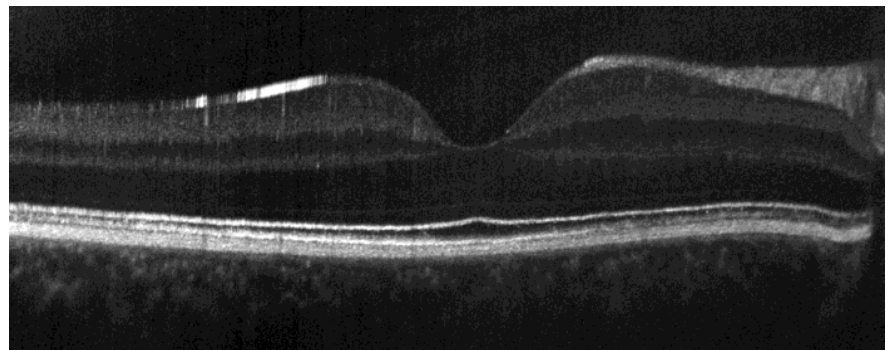

(a)

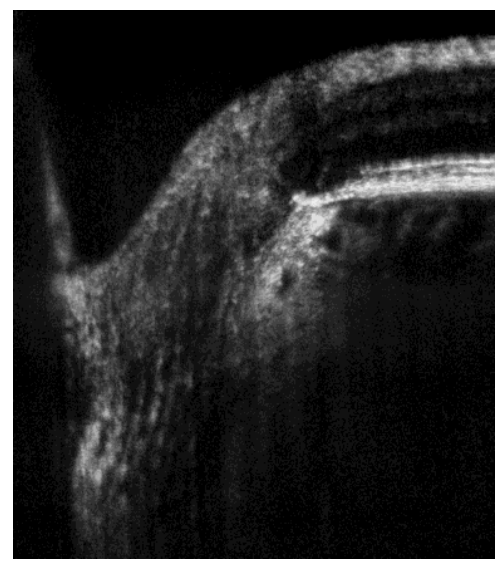

(b)

Fig. 23. OCT tomograms of the retina (a) and the optic nerve head (b) using the Ti:sapphire laser pumped by a frequency doubled DBR tapered diode laser.

\section{REFERENCES}

[1] A. Müller, S. Marschall, O. B. Jensen, J. Fricke, H. Wenzel, B. Sumpf, and P. E. Andersen, "Diode laser based light sources for biomedical applications," Laser Photon. Rev., p. DOI: 10.1002/lpor.201200051, Dec. 2012.

[2] D. Petring, R. Polzin, and M. Becker, "Applications," in in High Power Diode Lasers: Technology and Applications, F. Bachmann, P. Loosen, and R. Poprawe, Eds. Berlin: Springer, 2007, pp. 285533.

[3] P. Unger, "Introduction to Power Diode Lasers," in in High Power Diode Lasers: Technology and Applications, R. Diehl, Ed. Berlin: Springer, 2000, pp. 1-54.

[4] K. Suthamjariya and R. R. Anderson, "Lasers in Dermatology," in in Biomedical Photonics Handbook, T. Vo-Dinh, Ed. Boca Raton: CRC Press, 2003, pp. 1088-1127.

[5] L. V. Wang and H.-I. Wu, Biomedical Optics: Principles and Imaging. New Jersey: Wiley, 2007.

[6] W. Drexler and J. G. Fujimoto, Optical Coherence Tomography: Technology and Applications. Springer, 2008.

[7] S. Marschall, B. Sander, M. Mogensen, T. M. Jørgensen, and P. E. Andersen, "Optical coherence tomography-current technology and applications in clinical and biomedical research.," Anal. Bioanal. Chem., vol. 400, no. 9, pp. 2699-2720, Jul. 2011.

[8] Y. Yoshizumi, M. Adachi, Y. Enya, T. Kyono, S. Tokuyama, T. Sumitomo, K. Akita, T. Ikegami, M. Ueno, K. Katayama, and T. Nakamura, "Continuous-Wave Operation of $520 \mathrm{~nm}$ Green InGaNBased Laser Diodes on Semi-Polar $\{2021\}$ GaN Substrates," Appl. Phys. Express, vol. 2, no. 9, pp. 092101-1-3, Aug. 2009.

[9] A. Avramescu, T. Lermer, J. Müller, C. Eichler, G. Bruederl, M. Sabathil, S. Lutgen, and U. Strauss, "True Green Laser Diodes at $524 \mathrm{~nm}$ with $50 \mathrm{~mW}$ Continuous Wave Output Power on c-Plane GaN," Appl. Phys. Express, vol. 3, no. 6, pp. 061003-1-3, Jun. 2010.
T. Miyoshi, S. Masui, T. Okada, T. Yanamoto, T. Kozaki, S. Nagahama, and T. Mukai, " $510-515 \mathrm{~nm}$ InGaN-Based Green Laser Diodes on c-Plane GaN Substrate," Appl. Phys. Express, vol. 2, pp. 062201-1-3, May 2009.

[11] Y. Enya, Y. Yoshizumi, T. Kyono, K. Akita, M. Ueno, M. Adachi, T. Sumitomo, S. Tokuyama, T. Ikegami, K. Katayama, and T. Nakamura, "531 nm Green Lasing of InGaN Based Laser Diodes on Semi-Polar $\{2021\}$ Free-Standing GaN Substrates," Appl. Phys. Express, vol. 2, pp. 082101-1-3, Jul. 2009.

[12] S. Takagi, Y. Enya, T. Kyono, M. Adachi, Y. Yoshizumi, T. Sumitomo, Y. Yamanaka, T. Kumano, S. Tokuyama, K. Sumiyoshi, N. Saga, M. Ueno, K. Katayama, T. Ikegami, T. Nakamura, K. Yanashima, H. Nakajima, K. Tasai, K. Naganuma, N. Fuutagawa, Y. Takiguchi, T. Hamaguchi, and M. Ikeda, "High-Power (over 100 mW) Green Laser Diodes on Semipolar $\{2021\}$ GaN Substrates Operating at Wavelengths beyond $530 \mathrm{~nm}$," Appl. Phys. Express, vol. 5, pp. 082102-1-3, 2012.

[13] W. G. Zijlstra and A. Buursma, "Spectrophotometry of Hemoglobin: Absorption Spectra of Bovine Oxyhemoglobin, Deoxyhemoglobin, Carboxyhemoglobin, and Methemoglobin," Comp. Biochem. Physiol. Part B Biochem. Mol. Biol., vol. 118B, no. 4, pp. 743-749, 1997.

[14] W. R. Zipfel, R. M. Williams, and W. W. Webb, "Nonlinear magic: multiphoton microscopy in the biosciences.," Nat. Biotechnol., vol. 21, no. 11, pp. 1369-1377, Nov. 2003.

[15] E. E. Hoover and J. A. Squier, "Advances in multiphoton microscopy technology," Nat. Photonics, vol. 7, no. 2, pp. 93-101, Jan. 2013.

[16] K. Koenig and I. Riemann, "High-resolution multiphoton tomography of human skin with subcellular spatial resolution and picosecond time resolution," J. Biomed. Opt., vol. 8, no. 3, pp. 432439, 2003.

[17] W. Drexler, U. Morgner, F. X. Kärtner, C. Pitris, S. A. Boppart, X. D. Li, E. P. Ippen, and J. G. Fujimoto, "In vivo ultrahigh-resolution optical coherence tomography.," Opt. Lett., vol. 24, no. 17, pp. 1221-1223, Sep. 1999.

[18] A. Jechow, R. Menzel, K. Paschke, and G. Erbert, "Blue-green light generation using high brilliance edge emitting diode lasers," Laser Photon. Rev., vol. 4, no. 5, pp. 633-655, Sep. 2010.

[19] M. Chi, O. B. Jensen, J. Holm, C. Pedersen, P. E. Andersen, G. Erbert, B. Sumpf, and P. M. Petersen, "Tunable high-power narrowlinewidth semiconductor laser based on an external-cavity tapered amplifier," Opt. Express, vol. 13, no. 26, pp. 10589-10596, 2005.

[20] O. B. Jensen, B. Sumpf, G. Erbert, and P. M. Petersen, "Widely Tunable High-Power Tapered Diode Laser at 1060 nm," IEEE Photonics Technol. Lett., vol. 23, no. 21, pp. 1624-1626, 2011.

[21] G. Lucas-Leclin, D. Paboeuf, P. Georges, J. Holm, P. Andersen, B. Sumpf, and G. Erbert, "Wavelength stabilization of extended-cavity tapered lasers with volume Bragg gratings," Appl. Phys. B, vol. 91, no. 3-4, pp. 493-498, May 2008.

[22] R. J. Jones, S. Gupta, R. K. Jain, and J. N. Walpole, "Neardiffraction-limited high power ( $1 \mathrm{~W})$ single longitudinal mode $\mathrm{CW}$ diode laser tunable from 960 to 980 nm," Electron. Lett., vol. 31, no. 19 , pp. $1668-1669,1995$.

[23] S. Morgott, P. Chazan, M. Mikulla, M. Walther, R. Kiefer, J. Braunstein, and G. Weimann, "High-power near-diffraction-limited external cavity laser, tunable from 1030 to 1085 nm," Electron. Lett., vol. 34, no. 6, pp. 558-559, 1998.

[24] B. Sumpf, K.-H. Hasler, P. Adamiec, F. Bugge, F. Dittmar, Jö. Fricke, H. Wenzel, M. Zorn, G. Erbert, and G. Tränkle, "HighBrightness Quantum Well Tapered Lasers," IEEE J. Sel. Top. Quantum Electron., vol. 15, no. 3, pp. 1009-1020, 2009.

[25] C. Fiebig, G. Blume, C. Kaspari, D. Feise, J. Fricke, M. Matalla, W John, H. Wenzel, K. Paschke, and G. Erbert, "12 W high-brightness single-frequency DBR tapered diode laser," Electron. Lett., vol. 44, no. 21, pp. 1253-1255, 2008.

[26] H. Wenzel, K. Paschke, O. Brox, F. Bugge, J. Fricke, A. Ginolas, A Knauer, P. Ressel, and G. Erbert, "10 W continuous-wave monolithically integrated master-oscillator power amplifier," Electron. Lett., vol. 43, no. 3, pp. 160-161, 2007.

[27] O. B. Jensen, P. E. Andersen, B. Sumpf, K.-H. Hasler, G. Erbert, and P. M. Petersen, "1.5 W green light generation by single-pass second harmonic generation of a single-frequency tapered diode laser.," Opt. Express, vol. 17, no. 8, pp. 6532-6539, 2009. 
[28] C. Fiebig, J. Fricke, M. Uebernickel, D. Jedrzejczyk, A. Sahm, and K. Paschke, "Watt-Class Green-Emitting Laser Modules Using Direct Second Harmonic Generation of Diode Laser Radiation," Opt. Rev., vol. 19, no. 6, pp. 405-408, 2012.

[29] D. Jedrzejczyk, R. Güther, K. Paschke, W.-J. Jeong, H.-Y. Lee, and G. Erbert, "Efficient high-power frequency doubling of distributed Bragg reflector tapered laser radiation in a periodically poled $\mathrm{MgO}-$ doped lithium niobate planar waveguide.," Opt. Lett., vol. 36, no. 3, pp. 367-369, Feb. 2011.

[30] A. Müller, O. B. Jensen, K.-H. Hasler, B. Sumpf, G. Erbert, P. E. Andersen, and P. M. Petersen, "Efficient concept for generation of diffraction-limited green light by sum-frequency generation of spectrally combined tapered diode lasers.," Opt. Lett., vol. 37, no. 18, pp. 3753-3755, Sep. 2012.

[31] A. Müller, O. B. Jensen, A. Unterhuber, T. Le, A. Stingl, K.-H. Hasler, B. Sumpf, G. Erbert, P. E. Andersen, and P. M. Petersen, "Frequency-doubled DBR-tapered diode laser for direct pumping of Ti:sapphire lasers generating sub-20 fs pulses.," Opt. Express, vol. 19, no. 13, pp. 12156-12163, 2011.

[32] K. A. Fedorova, M. A. Cataluna, P. R. Battle, C. M. Kaleva, I. L. Krestnikov, D. A. Livshits, and E. U. Rafailov, "Orange light generation from a PPKTP waveguide end pumped by a cw quantum-dot tunable laser diode," Appl. Phys. B, vol. 103, no. 1, pp. 41-43, Nov. 2010.

[33] K. A. Fedorova, G. S. Sokolovskii, D. I. Nikitichev, P. R. Battle, I. L. Krestnikov, D. A. Livshits, and E. U. Rafailov, "Orange-to-red tunable picosecond pulses by frequency doubling in a diode-pumped PPKTP waveguide.," Opt. Lett., vol. 38, no. 15, pp. 2835-2837, Aug. 2013.

[34] W.-K. Lee, C. Y. Park, D.-H. Yu, S. E. Park, S.-B. Lee, and T. Y. Kwon, "Generation of 578-nm yellow light over $10 \mathrm{~mW}$ by second harmonic generation of an 1156-nm external-cavity diode laser.," Opt. Express, vol. 19, no. 18, pp. 17453-17461, Aug. 2011.

[35] P. A. Franken, A. E. Hill, C. W. Peters, and G. Weinreich, "Generation of optical harmonics," Phys. Rev. Lett., vol. 7, no. 4, pp. 118-120, 1961.

[36] M. Bass, P. A. Franken, A. E. Hill, C. W. Peters, and G. Weinreich, "Optical mixing," Phys. Rev. Lett., vol. 8, no. 1, pp. 18-19, 1962.

[37] R. C. Miller and A. Savage, "Harmonic Generation and Mixing of CaWO4:Nd3+ and Ruby Pulsed Laser Beams in Piezoelectric Crystals," Phys. Rev., vol. 128, no. 5, pp. 2175-2179, 1962.

[38] K. E. Niebuhr, "Generation of Laser Axial Mode Difference Frequencies in a Nonlinear Dielectric," Appl. Phys. Lett., vol. 2, no. 7, pp. 136-137, 1963.

[39] J. A. Giordmaine, "Mixing of light beams in crystals," Phys. Rev. Lett., vol. 8, no. 1, pp. 19-21, 1962.

[40] R. W. Boyd, Nonlinear Optics, Third Edit. Orlando: Academic Press, 2008.

[41] W. P. Risk, T. R. Gosnell, and A. V Nurmikko, Compact BlueGreen Lasers. Cambridge: Cambridge University Press, 2003.

[42] J. A. Armstrong, N. Bloembergen, J. Ducuing, and P. S. Pershan, "Interactions between light waves in a nonlinear dielectric," Phys. Rev., vol. 127, no. 6, pp. 1918-1939, 1962.

[43] M. M. Fejer, G. A. Magel, D. H. Jundt, and R. L. Byer, "Quasiphase-matched second harmonic generation: tuning and tolerances," IEEE J. Quantum Electron., vol. 28, no. 11, pp. 2631-2654, 1992.

[44] G. D. Boyd and D. A. Kleinman, "Parametric interaction of focused Gaussian light beams," J. Appl. Phys., vol. 39, no. 8, pp. 35973639, 1968.

[45] B. Beier, D. Woll, M. Scheidt, K. Boller, and R. Wallenstein, "Second harmonic generation of the output of an AlGaAs diode oscillator amplifier system in critically phase matched LiB3O5 and 3-BaB2O4," Appl. Phys. Lett., vol. 71, no. 3, pp. 315-317, 1997.

[46] J. D. Bhawalkar, Y. Mao, H. Po, a K. Goyal, P. Gavrilovic, Y. Conturie, and S. Singh, "High-power 390-nm laser source based on efficient frequency doubling of a tapered diode laser in an external resonant cavity.," Opt. Lett., vol. 24, no. 12, pp. 823-825, Jun. 1999.

[47] A. K. Goyal, J. D. Bhawalkar, Y. Conturie, P. Gavrilovic, Y. Mao, H. Po, and J. Guerra, "High beam quality of ultraviolet radiation generated through resonant enhanced frequency doubling of a diode laser," J. Opt. Soc. Am. B, vol. 16, no. 12, pp. 2207-2216, Dec. 1999.

[48] B. G. Klappauf, Y. Bidel, D. Wilkowski, T. Chanelière, and R. Kaiser, "Detailed study of an efficient blue laser source by secondharmonic generation in a semimonolithic cavity for the cooling of strontium atoms.," Appl. Opt., vol. 43, no. 12, pp. 2510-2527, Apr. 2004.

[49] J. H. Lundeman, O. B. Jensen, P. E. Andersen, S. AnderssonEngels, B. Sumpf, G. Erbert, and P. M. Petersen, "High power 404 $\mathrm{nm}$ source based on second harmonic generation in PPKTP of a tapered external feedback diode laser.," Opt. Express, vol. 16, no. 4, pp. 2486-2493, Feb. 2008

[50] J. H. Lundeman, O. B. Jensen, P. E. Andersen, and P. M. Petersen, "Threshold for strong thermal dephasing in periodically poled KTP in external cavity frequency doubling," Appl. Phys. B, vol. 96, no. 4, pp. 827-831, Aug. 2009.

[51] R. W. P. Drever, J. L. Hall, F. V. Kowalski, J. Hough, G. M. Ford, A. J. Munley, and H. Ward, "Laser phase and frequency stabilization using an optical resonator," Appl. Phys. B Photophysics Laser Chem., vol. 31, no. 2, pp. 97-105, Jun. 1983.

[52] T. W. Hansch and B. Couillaud, "Laser frequency stabilization by polarization spectroscopy of a reflecting reference cavity," Opt. Commun., vol. 35, no. 3, pp. 441-444, 1980.

[53] W. J. Kozlovsky, W. P. Risk, W. Lenth, B. G. Kim, G. L. Bona, H. Jaeckel, and D. J. Webb, "Blue light generation by resonatorenhanced frequency doubling of an extended-cavity diode laser," Appl. Phys. Lett., vol. 65, no. 5, pp. 525-527, 1994.

[54] X. G. Sun, G. W. Switzer, and J. L. Carlsten, "Blue light generation in an external ring cavity using both cavity and grating feedback," Appl. Phys. Lett., vol. 76, no. 8, pp. 955-957, 2000.

[55] D. Skoczowsky, A. Jechow, R. Menzel, K. Paschke, and G. Erbert, "Efficient second-harmonic generation using a semiconductor tapered amplifier in a coupled ring-resonator geometry.," Opt. Lett., vol. 35, no. 2, pp. 232-234, Jan. 2010.

[56] O. B. Jensen and P. M. Petersen, "Generation of single-frequency tunable green light in a coupled ring tapered diode laser cavity," Opt. Express, vol. 21, no. 5, pp. 6076-6081, Mar. 2013.

[57] A. Pietrzak, H. Wenzel, G. Erbert, and G. Tränkle, "High-power laser diodes emitting light above $1100 \mathrm{~nm}$ with a small vertical divergence angle of 13 degrees.," Opt. Lett., vol. 33, no. 19, pp. 2188-2190, Oct. 2008.

[58] P. Crump, G. Blume, K. Paschke, R. Staske, A. Pietrzak, U. Zeimer, S. Einfeldt, A. Ginolas, F. Bugge, K. Häusler, P. Ressel, H. Wenzel, and G. Erbert, "20W continuous wave reliable operation of $980 \mathrm{~nm}$ broad-area single emitter diode lasers with an aperture of $96 \mu \mathrm{m}$," in Proc. SPIE, 2009, pp. 719814-1-9.

[59] R. Platz, G. Erbert, W. Pittroff, M. Malchus, K. Vogel, and G. Tränkle, " $400 \mu \mathrm{m}$ stripe lasers for high-power fiber coupled pump modules," High Power Laser Sci. Eng., vol. 1, no. 1, pp. 60-67, 2013.

[60] M. Løbel, P. M. Petersen, and P. M. Johansen, "Single-mode operation of a laser-diode array with frequency-selective phaseconjugate feedback.," Opt. Lett., vol. 23, no. 11, pp. 825-827, Jun. 1998.

[61] S. Wolff, A. Rodionov, V. E. Sherstobitov, and H. Fouckhardt, "Fourier-Optical Transverse Mode Selection in External-Cavity Broad-Area Lasers : Experimental and Numerical Results," IEEE J. Quantum Electron., vol. 39, no. 3, pp. 448-458, 2003.

[62] E. Samsøe, P. E. Andersen, S. Andersson-Engels, and P. M. Petersen, "Improvement of spatial and temporal coherence of a broad area laser diode using an external-cavity design with double grating feedback.," Opt. Express, vol. 12, no. 4, pp. 609-616, Feb. 2004.

[63] A. Jechow, V. Raab, R. Menzel, M. Cenkier, S. Stry, and J. Sacher, " $1 \mathrm{~W}$ tunable near diffraction limited light from a broad area laser diode in an external cavity with a line width of $1.7 \mathrm{MHz}$," Opt. Commun., vol. 277, no. 1, pp. 161-165, Sep. 2007.

[64] J. N. Walpole, "Semiconductor amplifiers and lasers with tapered gain regions," Opt. Quantum Electron., vol. 28, pp. 623-645, 1996.

[65] M. Mikulla, "Tapered High-Power, High-Brightness Diode Lasers: Design and Performance," in in High Power Diode Lasers:

Technology and Applications, R. Diehl, Ed. Berlin: Springer, 2000, pp. 265-288.

[66] M. T. Kelemen, J. Weber, G. Kaufel, G. Bihlmann, R. Moritz, M. Mikulla, and G. Weimann, "Tapered diode lasers at $976 \mathrm{~nm}$ with 8 W nearly diffraction limited output power," Electron. Lett., vol. 41, no. 18, pp. 1011-1013, 2005.

[67] S. O’Brien, D. Mehuys, R. J. Lang, and D. F. Welch, "1 W CW single frequency, diffraction-limited unstable resonator 
semiconductor laser with distributed Bragg reflector mirrors," Electron. Lett., vol. 31, no. 3, pp. 203-205, 1995.

[68] R. Parke, D. F. Welch, A. Hardy, R. Lang, D. Mehuys, S. O. Brien, and D. Scifres, "2.0 W CW, Diffraction-Limited Operation of a Monolithically Integrated Master Oscillator Power Amplifier," IEEE Photonics Technol. Lett., vol. 5, no. 3, pp. 297-300, 1993.

[69] K.-H. Hasler, B. Sumpf, P. Adamiec, F. Bugge, J. Fricke, P. Ressel, H. Wenzel, G. Erbert, and G. Tränkle, "5-W DBR Tapered Lasers Emitting at $1060 \mathrm{~nm}$ With a Narrow Spectral Linewidth and a Nearly Diffraction-Limited Beam Quality," IEEE Photonics Technol. Lett., vol. 20, no. 19, pp. 1648-1650, 2008.

[70] R. Waarts, S. Sanders, R. Parke, D. Mehuys, R. Lang, S. O’Brien, K. Dzurko, D. Welch, and D. Scifres, "Frequency-doubled monolithic master oscillator power amplifier laser diode," IEEE Photonics Technol. Lett., vol. 5, no. 10, pp. 1122-1125, Oct. 1993.

[71] A. Jechow and R. Menzel, "Efficient blue light generation by frequency doubling of a broad-area diode laser in a compact external cavity," Appl. Phys. B, vol. 89, no. 4, pp. 507-511, Nov. 2007.

[72] A. Jechow, D. Skoczowsky, and R. Menzel, "100 $\mathrm{mW}$ high efficient single pass SHG at $488 \mathrm{~nm}$ of a single broad area laser diode with external cavity using a PPLN waveguide crystal.," Opt. Express, vol. 15, no. 11, pp. 6976-6981, May 2007.

[73] L. Goldberg, L. E. Busse, and D. Mehuys, "High power continuous wave blue light generation in $\mathrm{KNbO} 3$ using semiconductor amplifier seeded by a laser diode," Appl. Phys. Lett., vol. 63, no. 17, pp. 2327-2329, 1993.

[74] D. Fluck, T. Pliska, and P. Günter, "Compact $10 \mathrm{~mW}$ all solid state $491 \mathrm{~nm}$ laser based on frequency doubling a MOPA laser diode," Opt. Commun., vol. 123, pp. 624-628, 1996.

[75] M. Maiwald, S. Schwertfeger, R. Güther, B. Sumpf, K. Paschke, C. Dzionk, G. Erbert, and G. Tränkle, " $600 \mathrm{~mW}$ optical output power at $488 \mathrm{~nm}$ by use of a high-power hybrid laser diode system and a periodically poled $\mathrm{MgO}: \mathrm{LiNbO} 3$ bulk crystal.," Opt. Lett., vol. 31, no. 6, pp. 802-804, Mar. 2006.

[76] C. Fiebig, A. Sahm, M. Uebernickel, G. Blume, B. Eppich, K. Paschke, and G. Erbert, "Compact second-harmonic generation laser module with $1 \mathrm{~W}$ optical output power at $490 \mathrm{~nm}$.," Opt. Express, vol. 17, no. 25, pp. 22785-22790, Dec. 2009.

[77] B. Sumpf, K.-H. Hasler, P. Adamiec, F. Bugge, J. Fricke, P. Ressel, H. Wenzel, G. Erbert, and G. Tränkle, "1060 nm DBR tapered lasers with $12 \mathrm{~W}$ output power and a nearly diffraction limited beam quality," in Proc. SPIE, 2009, vol. 7230, p. 72301E-72301E-8.

[78] M. Uebernickel, R. Güther, G. Blume, C. Fiebig, K. Paschke, and G. Erbert, "Study of the properties of the SHG with diode lasers," Appl. Phys. B, vol. 99, no. 3, pp. 457-464, Mar. 2010.

[79] K. Paschke, C. Fiebig, D. Feise, J. Fricke, C. Kaspari, G. Blume, H. Wenzel, and G. Erbert, "High-power single-frequency operation of a DBR tapered laser," in IEEE 21 st International Semiconductor Laser Conference, 2008. ISLC 2008., 2008, vol. 6184, no. 2006, pp. 131-132.

[80] K. Paschke, C. Fiebig, G. Blume, F. Bugge, J. Fricke, and G. Erbert, "1120nm highly brilliant laser sources for SHG-modules in bioanalytics and spectroscopy," in Proc. SPIE, 2013, vol. 8640, pp. $86401 \mathrm{~J}-1-8$.

[81] J. M. Yarborough, J. Falk, and C. B. Hitz, "Enhancement of Optical Second Harmonic Generation By Utilizing the Dispersion of Air," Appl. Phys. Lett., vol. 18, no. 3, pp. 70-73, 1971.

[82] S. Spiekermann, F. Laurell, V. Pasiskevicius, H. Karlsson, and I. Freitag, "Optimizing non-resonant frequency conversion in periodically poled media," Appl. Phys. B, vol. 79, no. 2, pp. 211219, May 2004.

[83] D. Fluck and P. Günter, "Efficient second-harmonic generation by lens wave-guiding in $\mathrm{KNbO} 3$ crystals," Opt. Commun., vol. 147, no. 4-6, pp. 305-308, 1998.

[84] S. Vasilyev, A. Nevsky, I. Ernsting, M. Hansen, J. Shen, and S. Schiller, "Compact all-solid-state continuous-wave single-frequency UV source with frequency stabilization for laser cooling of $\mathrm{Be}+$ ions," Appl. Phys. B, vol. 103, no. 1, pp. 27-33, Feb. 2011.

[85] G. K. Samanta, S. C. Kumar, K. Devi, and M. Ebrahim-Zadeh, "Multicrystal, continuous-wave, single-pass second-harmonic generation with $56 \%$ efficiency," Opt. Lett., vol. 35, no. 20, pp. 3513-3515, 2010.

[86] S. Chiow, T. Kovachy, J. M. Hogan, and M. A. Kasevich, "Generation of $43 \mathrm{~W}$ of quasi-continuous $780 \mathrm{~nm}$ laser light via high-efficiency, single-pass frequency doubling in periodically poled lithium niobate crystals.," Opt. Lett., vol. 37, no. 18, pp. 3861-3863, Sep. 2012.

[87] A. K. Hansen, O. B. Jensen, P. E. Andersen, and P. M. Petersen, Opt. Lett., Manuscript under preparation, 2013.

[88] J. C. Bienfang, C. A. Denman, B. W. Grime, P. D. Hillman, G. T. Moore, and J. M. Telle, " $20 \mathrm{~W}$ of continuous-wave sodium D2 resonance radiation from sum-frequency generation with injectionlocked lasers.," Opt. Lett., vol. 28, no. 22, pp. 2219-2221, Nov. 2003.

[89] N. Saito, K. Akagawa, M. Ito, A. Takazawa, Y. Hayano, Y. Saito, M. Ito, H. Takami, M. Iye, and S. Wada, "Sodium D2 resonance radiation in single-pass sum-frequency generation with actively mode-locked Nd:YAG lasers.," Opt. Lett., vol. 32, no. 14, pp. 19651967, Jul. 2007.

[90] J. Yue, C.-Y. She, B. P. Williams, J. D. Vance, P. E. Acott, and T. D. Kawahara, "Continuous-wave sodium D2 resonance radiation generated in single-pass sum-frequency generation with periodically poled lithium niobate.," Opt. Lett., vol. 34, no. 7, pp. 1093-1095, Apr. 2009.

[91] J.-C. Baumert, F. M. Schellenberg, W. Lenth, W. P. Risk, and G. C. Bjorklund, "Generation of blue cw coherent radiation by sum frequency mixing in KTiOPO4," Appl. Phys. Lett., vol. 51, no. 26, pp. 2192-2194, 1987.

[92] W. P. Risk, J.-C. Baumert, G. C. Bjorklund, F. M. Schellenberg, and W. Lenth, "Generation of blue light by intracavity frequency mixing of the laser and pump radiation of a miniature neodymium:yttrium aluminum garnet laser," Appl. Phys. Lett., vol. 52, no. 2, pp. 85-87, 1988.

[93] P. N. Kean and G. J. Dixon, "Efficient sum-frequency upconversion in a resonantly pumped Nd:YAG laser.," Opt. Lett., vol. 17, no. 2, pp. 127-129, Jan. 1992.

[94] K. P. Sørensen, P. Tidemand-Lichtenberg, and C. Pedersen, "Efficient near diffraction limited blue light source by sumfrequency mixing of a BAL and a solid-state laser," Laser Phys. Lett., vol. 8, no. 3, pp. 209-213, Mar. 2011.

[95] B. Thestrup, M. Chi, B. Sass, and P. M. Petersen, "High brightness laser source based on polarization coupling of two diode lasers with asymmetric feedback," Appl. Phys. Lett., vol. 82, no. 5, pp. 680$682,2003$.

[96] O. Andrusyak, V. Smirnov, G. Venus, and L. Glebov, "Beam combining of lasers with high spectral density using volume Bragg gratings," Opt. Commun., vol. 282, no. 13, pp. 2560-2563, Jul. 2009.

[97] O. Andrusyak, V. Smirnov, G. Venus, V. Rotar, and L. Glebov, "Spectral Combining and Coherent Coupling of Lasers by Volume Bragg Gratings," IEEE J. Sel. Top. Quantum Electron., vol. 15, no. 2, pp. 344-353, 2009.

[98] A. Müller, D. Vijayakumar, O. B. Jensen, K.-H. Hasler, B. Sumpf, G. Erbert, P. E. Andersen, and P. M. Petersen, " $16 \mathrm{~W}$ output power by high-efficient spectral beam combining of DBR-tapered diode lasers.," Opt. Express, vol. 19, no. 2, pp. 1228-1235, 2011.

[99] E. Karamehmedović, C. Pedersen, O. B. Jensen, and P. TidemandLichtenberg, "Nonlinear beam clean-up using resonantly enhanced sum-frequency mixing," Appl. Phys. B, vol. 96, no. 2-3, pp. 409413, May 2009.

[100] V. Daneu, A. Sanchez, T. Y. Fan, H. K. Choi, G. W. Turner, and C. C. Cook, "Spectral beam combining of a broad-stripe diode laser array in an external cavity.," Opt. Lett., vol. 25, no. 6, pp. 405-407, Mar. 2000.

[101] B. Chann, R. K. Huang, L. J. Missaggia, C. T. Harris, Z. L. Liau, A. K. Goyal, J. P. Donnelly, T. Y. Fan, A. Sanchez-Rubio, and G. W. Turner, "Near-diffraction-limited diode laser arrays by wavelength beam combining.," Opt. Lett., vol. 30, no. 16, pp. 2104-2106, Aug. 2005.

[102] A. Jechow, V. Raab, and R. Menzel, "High cw power using an external cavity for spectral beam combining of diode laser-bar emission.," Appl. Opt., vol. 45, no. 15, pp. 3545-3547, May 2006.

[103] D. Vijayakumar, O. B. Jensen, and B. Thestrup, "980 nm high brightness external cavity broad area diode laser bar.," Opt. Express, vol. 17, no. 7, pp. 5684-5690, 2009.

[104] D. Vijayakumar, O. B. Jensen, R. Ostendorf, T. Westphalen, and B. Thestrup, "Spectral beam combining of a $980 \mathrm{~nm}$ tapered diode laser bar.," Opt. Express, vol. 18, no. 2, pp. 893-898, 2010. 
[105] E. Hecht, Optics Fourth Edition. San Francisco: Addison-Wesley, 2002.

[106] P. F. Moulton, "Spectroscopic and laser characteristics of Ti:A1203," J. Opt. Soc. Am. B, vol. 3, no. 1, pp. 125-133, 1986.

[107] K. F. Wall and A. Sanchez, "Titanium Sapphire Lasers," Lincolm Lab. J., vol. 3, no. 3, pp. 447-462, 1990.

[108] A. Unterhuber, B. Povazay, B. Hermann, H. Sattmann, W. Drexler, V. Yakovlev, G. Tempea, C. Schubert, E. M. Anger, P. K. Ahnelt, M. Stur, J. E. Morgan, A. Cowey, G. Jung, T. Le, and A. Stingl, "Compact, low-cost Ti:Al2O3 laser for in vivo ultrahigh-resolution optical coherence tomography.," Opt. Lett., vol. 28, no. 11, pp. 905907, Jun. 2003.

[109] A. Unterhuber, B. Povazay, A. Müller, O. B. Jensen, T. Otto, I. Boettcher, M. Duelk, R. Kessler, R. Engelhardt, M. Esmaeelpour, T. Le, P. E. Andersen, C. Velez, G. Zinser, and W. Drexler,

"Simultaneous dual wavelengths eye tracked ultrahigh resolution retinal and choroidal OCT," Opt. Lett., Accepted, 2013.

[110] T. Baer, "Large-amplitude fluctuations due to longitudinal mode coupling in diode-pumped intracavity-doubled Nd:YAG lasers," $J$. Opt. Soc. Am. B, vol. 3, no. 9, pp. 1175-1180, Sep. 1986.

[111] P. W. Roth, A. J. Maclean, D. Burns, and A. J. Kemp, "Directly diode-laser-pumped Ti:sapphire laser.," Opt. Lett., vol. 34, no. 21, pp. 3334-3336, Nov. 2009.

[112] P. W. Roth, D. Burns, and A. J. Kemp, "Power scaling of a directly diode-laser-pumped Ti:sapphire laser.," Opt. Express, vol. 20, no. 18, pp. 20629-20634, Aug. 2012.

[113] P. W. Roth, A. J. Maclean, D. Burns, and A. J. Kemp, "Direct diode-laser pumping of a mode-locked Ti:sapphire laser.," Opt. Lett., vol. 36, no. 2, pp. 304-306, Jan. 2011.

[114] C. G. Durfee, T. Storz, J. Garlick, S. Hill, J. a Squier, M. Kirchner, G. Taft, K. Shea, H. Kapteyn, M. Murnane, and S. Backus, "Direct diode-pumped Kerr-lens mode-locked Ti:sapphire laser.," Opt. Express, vol. 20, no. 13, pp. 13677-13683, Jun. 2012. 


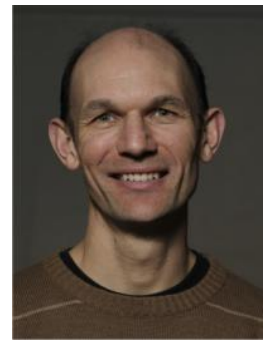

Ole Bjarlin Jensen received his M.Sc. in applied physics from the Technical University of Denmark (DTU) in 1999 and the Ph.D. degree in optics from DTU in 2002. From 2002 to 2003, he was development engineer at ASAH Medicos A/S. In 2004 he joined the Ris $\varnothing$ National Laboratory as Post doc and in 2007 the Technical University of Denmark as researcher. In 2011 he became senior researcher at the department of photonics engineering, DTU Fotonik. His current research interests include diode lasers, solid state lasers and nonlinear frequency conversion as well as biomedical applications of laser systems.

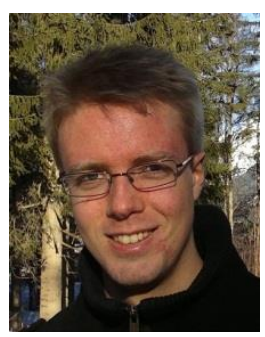

Anders Kragh Hansen received his M.Sc. and Ph.D. degrees in Physics in 2010 and 2013, respectively, working on laser cooling of molecular ions at the Ion Trap Group at Aarhus University. In 2013, he joined DTU Fotonik at the Technical University of Denmark as a Post doc, where he is currently working on research and development of laser modules involving nonlinear frequency conversion with laser diodes as sources. Biophotonics applications are of special importance to this work.

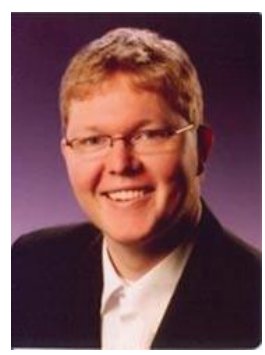

André Müller received his M. Eng. degree in Applied Physics/Medical Engineering from the University of Applied Sciences in Berlin in 2008. In his thesis he focused on the development of compact diode laser systems for spectroscopic applications. In 2009 he joined the group of Paul Michael Petersen at the Technical University of Denmark (DTU). As a PhD student at DTU from 2010 to 2013 he has been working on suitable methods for power scaling of frequency converted diode lasers. $\mathrm{He}$ is currently with Ferdinand-Braun-Institut in Berlin working on high-brightness diode lasers and diode laser systems for spectroscopic applications.

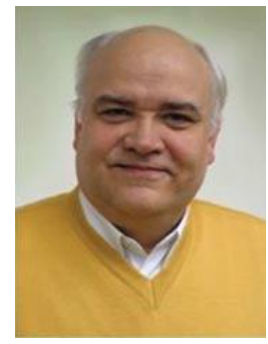

Bernd Sumpf received his Diplom in Physics in 1981 and the Ph.D. degree in 1987 from the Humboldt-Universität zu Berlin for his work on lead salt diode lasers for spectroscopic applications. From 1993 till 1997 he worked at the Technische Universität Berlin on highresolution spectroscopy and non-linear optics and received the postdoctoral lecture qualification in 1997. Since 2000 he is at the Ferdinand-Braun-Institut Berlin. His current research topics are high-brightness diode lasers and diode lasers for sensory applications and RAMAN spectroscopy.

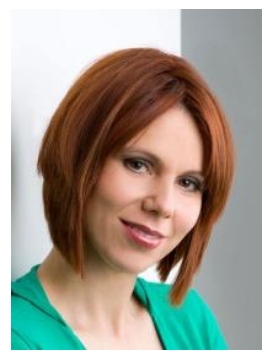

Angelika Unterhuber received her M.Sc. in physics from the Vienna University of Technology in 1998. From 1998 to 2002 she was product manager for ultrafast lasers at Femtolasers Produktions $\mathrm{GmbH}$. In 2006 she received the Pd.D. degree in Electrical Engineering and joined the School of Optometry and Vision Sciences, Cardiff. She returned to Vienna with a Marie Curie Fellowship in 2009. Now she works at the Medical University of Vienna where her current research interests are focused on development of novel light sources and their biomedical application especially in the field of OCT and nonlinear imaging.

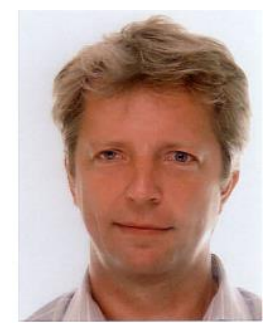

Wolfgang Drexler was a Full Professor of Biomedical Imaging at the School of Optometry and Vision Sciences at Cardiff University, Wales, UK from 2006 to 2009. Since October 2009 he is a Full Professor of Medical Physics and the Head of the Center for Medical Physics and Biomedical Engineering at the Medical University of Vienna, Austria, Honorary Distinguished Professor at Cardiff University and is also Director of the Christian Doppler Laboratory. He is a member of the Austrian Academy of Science and has published more than 150 publications (including Nature Medicine and PNAS).

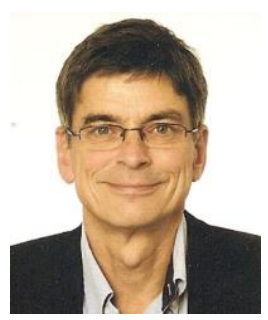

Paul Michael Petersen received the M.Sc. degree in engineering and the Ph.D. degree in physics from the Technical University of Denmark in 1983 and 1986, respectively. Prof. Petersen has more than 25 years of research experience in laser physics, nonlinear optics, and optical measuring techniques and he has headed several collaborative research projects within laser physics and LED lighting. From 2002 to 2012 he was adjunct professor in Optics at the Niels Bohr Institute, Copenhagen University. In 2011 P. M. Petersen was appointed Full Professor in New Light Sources at the Technical University of Denmark.

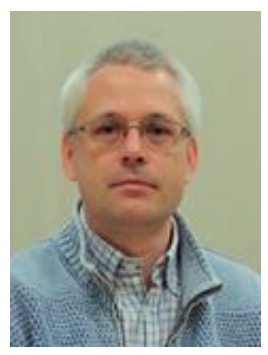

Peter E. Andersen received his MSc.E.E. in 1991 and his Ph.D. in 1994 from the Technical University of Denmark. Dr. Andersen is Senior Scientist and Research Professor at the Technical University of Denmark, where he leads the research within biomedical optics. He has more than 15 years of research experience with light sources for biomedical optics and optical coherence tomography systems and their application. Dr. Andersen has coordinated several European research programs. He has authored or co-authored more than 125 scientific publications in the above-mentioned fields, respectively. Dr. Andersen is appointed Deputy Editor of Optics Letters, editorial board member of Journal of Biomedical Optics, and editorial board member of Journal of Biophotonics, respectively. 